\title{
DEFORMATIONS OF $G_{2}$-STRUCTURES WITH TORSION*
}

\author{
SERGEY GRIGORIAN ${ }^{\dagger}$
}

\begin{abstract}
We consider non-infinitesimal deformations of $G_{2}$-structures on 7-dimensional manifolds and derive an exact expression for the torsion of the deformed $G_{2}$-structure. We then specialize to a case when the deformation is defined by a vector $v$ and we explicitly derive the expressions for the different torsion components of the new $G_{2}$-structure in terms of the old torsion components and derivatives of $v$. In particular this gives a set of differential equations for the vector $v$ which have to be satisfied for a transition between $G_{2}$-structures with particular torsions. For some specific torsion classes we find that these equations have no solutions.
\end{abstract}

Key words. G2-structures, deformations, holonomy.

AMS subject classifications. 53C10, 53C25, 53C29.

1. Introduction. Seven-dimensional manifolds with $G_{2}$-structure have been studied for more than 40 years. Already in 1969, Alfred Gray studied vector cross products on manifolds [8], which on 7-manifolds do actually correspond to $G_{2^{-}}$ structures. Later on, Fernández and Gray classified the possible torsion classes of $G_{2}$-structures [6]. It is well-known that a 7 -manifolds admits a $G_{2}$-structure if and only if the first two Stiefel-Whitney classes $w_{1}$ and $w_{2}$ vanish $[6,7]$. Alternatively, a 7 -manifold admits a $G_{2}$-structure if and only if it is orientable and admits a spin structure. A very important special case of a $G_{2}$-structure is when the torsion vanishes. This implies that the Riemannian holonomy group lies in $G_{2}$. In Section 2 we give a more precise definition and an overview of the properties of $G_{2}$-structures.

Suppose we are given a 7 -manifold that admits a $G_{2}$-structure, we can ask the question - which torsion classes of $G_{2}$-structures exist on it? This is of course a very difficult question, and it is still not clear how to approach this. However, we could start with some given $G_{2}$-structure, deform it and then require that the new $G_{2^{-}}$ structure lies in some particular torsion class. This is precisely what we attempt in this paper. First, in Section 4, we consider non-infinitesimal deformations of the $G_{2^{-}}$ structure 3-form and obtain closed expressions for the related quantities - the volume form, the metric and the dual 4-form. Remarkably, the deformed metric and 4-form are expressible as polynomials in the deformation form, multiplied by an integer power of the ratio of the old and deformed volume forms. This ratio of the volume forms can the be further expressed as a polynomial in the deformation form raised to a rational power. Using these results, in Section 5 we then derive an expression for the $G_{2}$-structure torsion for a general (non-infinitesimal) deformation, and then in Section 6 , we specialize to a particular type of deformation - deformations that are defined by a vector (that is, the $G_{2}$ invariant 3 -form is deformed by a 3 -form lying in the 7-dimensional component of $\Lambda^{3}$ ). In this case, we obtain an explicit expression for the torsion of the deformed $G_{2}$-structure in terms of the old $G_{2}$-structure, its torsion and the vector which defines the deformation. We then proceed to show that a noninfinitesimal deformation of this type takes a torsion-free $G_{2}$-structure to another torsion-free $G_{2}$-structure if and only if the vector that defines the deformation is parallel. Moreover we also show that on closed, compact manifolds there are no such

\footnotetext{
*Received August 9, 2013; accepted for publication October 6, 2014.

†School of Mathematical and Statistical Sciences, University of Texas Rio Grande Valley, $1201 \mathrm{~W}$ University Drive, Edinburg, TX 78539, USA (sergey.grigorian@utrgv.edu).
} 
deformations from strict torsion classes $W_{1}, W_{7}, W_{1} \oplus W_{7}$ to the vanishing torsion class $W_{0}$.

Such deformations of $G_{2}$-structures have been first considered by Karigiannis in [13], where he wrote down the deformed metric and Hodge star operation, and indeed asked the question whether it is possible to deform a $G_{2}$-structure to a strictly smaller torsion class. This paper aims to give a partial answer to this question. Here we are mainly concerned with non-infinitesimal deformations, but infinitesimal deformations and flows of $G_{2}$-structures, and in particular properties of the moduli space of manifolds with $G_{2}$ holonomy have also been studied by Karigiannis [13, 14], Karigiannis and Leung [15] and by Grigorian and Yau [9, 10].

2. $G_{2}$-structures. The 14-dimensional group $G_{2}$ is the smallest of the five exceptional Lie groups and is closely related to the octonions. In particular, $G_{2}$ can be defined as the automorphism group of the octonion algebra. We can use octonion multiplication on imaginary octonions to define a vector cross product on $V=\mathbb{R}^{7}$. Let $a, b \in \mathbb{R}^{7}$, then identifying $a$ and $b$ with imaginary octonions, we define the cross product as

$$
a \times b=\operatorname{Im}(a b)
$$

where $a b$ is now the octonionic product of $a$ and $b$.

Moreover, the Euclidean inner product on $V$ can also be defined in terms of octonion multiplication. The group that preserves the vector cross product is precisely $G_{2}$ and since it preserves the inner product as well, we can see that it is a subgroup of $O(7)$. It can further be shown that $G_{2}$ is a connected group, and is in fact a subgroup of $S O(7)$. For more on the relationship between octonions and $G_{2}$, see $[1,9]$.The structure constants of the vector cross product define a particular 3 -form on $\mathbb{R}^{7}$, hence $G_{2}$ can alternatively be defined in the following way.

Definition 2.1. Let $\left(e^{1}, e^{2}, \ldots, e^{7}\right)$ be a basis for $V^{*}$, and denote $e^{i} \wedge e^{j} \wedge e^{k}$ by $e^{i j k}$. Then define $\varphi_{0}$ to be the 3 -form on $\mathbb{R}^{7}$ given by

$$
\varphi_{0}=e^{123}+e^{145}+e^{167}+e^{246}-e^{257}-e^{347}-e^{356} .
$$

Then $G_{2}$ is defined as the subgroup of $G L(7, \mathbb{R})$ which preserves $\varphi_{0}$.

Suppose for some 3 -form $\varphi$ on $V$ we define a bilinear form by

$$
\left.\left.B_{\varphi}(u, v)=\frac{1}{6}(u\lrcorner \varphi\right) \wedge(v\lrcorner \varphi\right) \wedge \varphi
$$

Here the symbol $\lrcorner$ denotes contraction of a vector with the differential form:

$$
(u\lrcorner \varphi)_{m n}=u^{a} \varphi_{a m n} .
$$

Note that we will also use this symbol for contractions of differential forms using the metric. So for a $p$-form $\alpha$ and a $(p+q)$-form $\beta$, for $q \geq 0$,

$$
(\alpha\lrcorner \beta)_{b_{1} \ldots b_{q}}=\alpha^{a_{1} \ldots a_{p}} \beta_{a_{1} \ldots a_{p} b_{1} \ldots b_{q}}
$$

where the indices on $\alpha$ are raised using the metric.

Following Hitchin ([11]), $B_{\varphi}$ is a symmetric bilinear form on $V$ with values in the one-dimensional space $\Lambda^{7} V^{*}$. Hence it defines a linear map $K_{\varphi}: V \longrightarrow V^{*} \times \Lambda^{7} V^{*}$. Then taking the determinant we get $\operatorname{det} K_{\varphi} \in\left(\Lambda^{7} V^{*}\right)^{\otimes 9}$, so if this does not vanish, 
we choose a positive root - $\left(\operatorname{det} K_{\varphi}\right)^{\frac{1}{9}} \in \Lambda^{7} V^{*}$. Then we obtain an inner product on $V$

$$
g_{\varphi}(u, v)=B_{\varphi}(u, v)\left(\operatorname{det} K_{\varphi}\right)^{-\frac{1}{9}}
$$

and the volume form of this inner product is then $\left(\operatorname{det} K_{\varphi}\right)^{\frac{1}{9}}$. In components we can rewrite this as

$$
\left(g_{\varphi}\right)_{a b}=(\operatorname{det} s)^{-\frac{1}{9}} s_{a b}
$$

with

$$
s_{a b}=\frac{1}{144} \varphi_{a m n} \varphi_{b p q} \varphi_{r s t} \hat{\varepsilon}^{m n p q r s t}
$$

where $\hat{\varepsilon}^{\text {mnpqrst }}$ is the alternating symbol with $\hat{\varepsilon}^{12 \ldots 7}=+1$.

Applying (2.2) to $\varphi_{0}$ as defined by (2.1), we recover the standard Euclidean metric on $V$ :

$$
g_{0}=\left(e^{1}\right)^{2}+\ldots+\left(e^{7}\right)^{2} .
$$

As we know, the stabilizer of $\varphi_{0}$ in $G L(7, \mathbb{R})$ is $G_{2}$, which is 14-dimensional. Since $G L(7, \mathbb{R})$ is 49 -dimensional, we find that the orbit of $\varphi_{0}$ in $\Lambda^{3} V^{*}$ has dimension $49-14=35=\operatorname{dim} \Lambda^{3} V^{*}$. Hence the orbit of $\varphi_{0}$ is an open subset $\Lambda_{+}^{3} \subset \Lambda^{3} V^{*}$.

Definition 2.2. Let $V$ be a 7-dimensional real vector space. Then a 3-form $\varphi$ is said to be positive if it lies in the $G L(7, \mathbb{R})$ orbit of $\varphi_{0}$.

In fact, in $\Lambda^{3} V^{*}$ there are two open orbits of $G L(7, \mathbb{R})[2]$. The second open orbit consists of 3 -forms for which the metric defined by $(2.4)$ has indefinite signature $(4,3)$, and the corresponding stabilizer is the so-called split $G_{2}$. The 3 -form that it stabilizes can be obtained by changing the minus signs to plus signs in the expression (2.1) for $\varphi_{0}$. The existence of these open orbits gives a notion of a non-degenerate 3 -form on $V$ - that is, a 3-form which lies in one of the open orbits [11]. Moreover, it turns out that non-degeneracy of a 3 -form is equivalent to non-degeneracy of the corresponding metric. Thus if the determinant of the metric (2.5), or equivalently $\operatorname{det}\left(s_{a b}\right)$ for $s_{a b}$ in (2.6), is non-zero, then the 3 -form is in one of the open orbits. If moreover, the metric is positive-definite, then the 3 -form is positive.

Now, given a $n$-dimensional manifold $M$, a $G$-structure on $M$ for some Lie subgroup $G$ of $G L(n, \mathbb{R})$ is a reduction of the frame bundle $F$ over $M$ to a principal subbundle $P$ with fibre $G$. The concept of a $G$-structure gives a convenient way of encoding different geometric structures. For example, an $O(n)$-structure is a reduction of the frame bundle to a subbundle with fibre $O(n)$. This defines an orthonormal frame at each point at $M$ and thus we can define a Riemannian metric on $M$. Hence there is a 1-1 correspondence between $O(n)$-structures and Riemannian metrics. Similarly, an almost complex structure on a $2 m$-dimensional manifold $M$ is equivalent to a $G L(m, \mathbb{C})$-structure.

A $G_{2}$-structure is then a reduction of the frame bundle on a 7 -dimensional manifold $M$ to a $G_{2}$ principal subbundle. It turns out that there is a 1-1 correspondence between $G_{2}$-structures and positive 3 -forms on the manifold. Define the bundle of positive 3 -forms on $M$ as the subset of 3-forms $\varphi$ in $\Lambda^{3} T^{*} M$ such that for every point $p$ in $M,\left.\varphi\right|_{p} \in \Lambda^{3} T_{p}^{*} M$ is a positive 3 -form in the sense of Definition 2.2. Using the 
$G_{2}$ principal bundle we can then define a positive 3 -form $\varphi$ on the whole manifold. Conversely, suppose we are given a positive 3 -form. Then at each point $p$ the subset of $G L(7, \mathbb{R})$ that identifies $\left.\varphi\right|_{p}$ with $\varphi_{0}$ is isomorphic to $G_{2}$. Over the whole manifold this will be a subset of the frame bundle $F$, and it is easy to show that this does give a principal subbundle with fibre $G_{2}$, and hence a $G_{2}$-structure.

Once we have a $G_{2}$-structure, since $G_{2}$ is a subgroup of $S O(7)$, we can also define a Riemannian metric. More concretely, if $\varphi$ is the 3-form which defines the $G_{2}$ structure, then using (2.4) we can define a corresponding metric $g$. Following Joyce ([12]), we will adopt the following abuse of notation

DeFINITION 2.3. Let $M$ be an oriented 7 -manifold. The pair $(\varphi, g)$ for a positive 3 -form $\varphi$ and corresponding metric $g$ will be referred to as a $G_{2}$-structure.

Since a $G_{2}$-structure defines a metric, it also defines a Hodge star. Thus we can construct another $G_{2}$-invariant object - the 4 -form $* \varphi$. Since the Hodge star is defined by the metric, which in turn is defined by $\varphi$, the 4 -form $* \varphi$ depends non-linearly on $\varphi$. For convenience we will usually denote $* \varphi$ by $\psi$. On $\mathbb{R}^{7}$, when $\varphi$ is given by its canonical form $\varphi_{0}(2.1), \psi$ takes the following canonical form

$$
\psi_{0}=e^{4567}+e^{2367}+e^{2345}+e^{1357}-e^{1346}-e^{1256}-e^{1247} .
$$

By considering the canonical forms $\varphi_{0}$ and $\psi_{0}$, we can write down various contraction identities for a $G_{2}$-structure $(\varphi, g)$ and its corresponding 4-form $\psi[3,10,14]$.

Proposition 2.4. The 3-form $\varphi$ and the corresponding 4-form $\psi$ satisfy the following identities:

$$
\begin{aligned}
\varphi_{a b c} \varphi_{m n}{ }^{c} & =g_{a m} g_{b n}-g_{a n} g_{b m}+\psi_{a b m n} \\
\varphi_{a b c} \psi_{m n p}{ }^{c} & =3\left(g_{a[m} \varphi_{n p] b}-g_{b[m} \varphi_{n p] a}\right) \\
\psi_{a b c d} \psi^{m n p q} & =24 \delta_{a}^{[m} \delta_{b}^{n} \delta_{c}^{p} \delta_{d}^{q]}+72 \psi_{[a b}{ }^{[m n} \delta_{c}^{p} \delta_{d]}^{q]}-16 \varphi_{[a b c} \varphi^{[m n p} \delta_{d]}^{q]}
\end{aligned}
$$

where $\left[\begin{array}{ll}m & n\end{array}\right]$ denotes antisymmetrization of indices and $\delta_{a}^{b}$ is the Kronecker delta, with $\delta_{b}^{a}=1$ if $a=b$ and 0 otherwise.

The above identities can be of course further contracted - the details can be found in $[10,14]$. These identities and their contractions are crucial whenever any calculations involving $\varphi$ and $\psi$ have to be done.

For a general $G$-structure, the spaces of $p$-forms decompose according to irreducible representations of $G$. Given a $G_{2}$-structure, we have the following decomposition of $p$-forms:

$$
\begin{aligned}
\Lambda^{1} & =\Lambda_{7}^{1} \\
\Lambda^{2} & =\Lambda_{7}^{2} \oplus \Lambda_{14}^{2} \\
\Lambda^{3} & =\Lambda_{1}^{3} \oplus \Lambda_{7}^{3} \oplus \Lambda_{27}^{3} \\
\Lambda^{4} & =\Lambda_{1}^{4} \oplus \Lambda_{7}^{4} \oplus \Lambda_{27}^{4} \\
\Lambda^{5} & =\Lambda_{7}^{5} \oplus \Lambda_{14}^{5} \\
\Lambda^{6} & =\Lambda_{7}^{6}
\end{aligned}
$$

The subscripts denote the dimension of the representation and components which correspond to the same representation are isomorphic to each other. We have the following characterization of the various components $[2,3]$ : 
Proposition 2.5. Let $M$ be a 7-manifold with a $G_{2}$-structure $(\varphi, g)$. Then the components of spaces of 2-, 3-, 4-, and 5-forms are given by

$$
\begin{aligned}
\Lambda_{7}^{2} & \left.=\{\alpha\lrcorner \varphi: \alpha \in \Lambda_{7}^{1}\right\} \\
\Lambda_{14}^{2} & \left.=\left\{\omega \in \Lambda^{2}:\left(\omega_{a b}\right) \in \mathfrak{g}_{2}\right\}=\left\{\omega \in \Lambda^{2}: \omega\right\lrcorner \varphi=0\right\} \\
\Lambda_{1}^{3} & =\left\{f \varphi: f \in C^{\infty}(M)\right\} \\
\Lambda_{7}^{3} & \left.=\{\alpha\lrcorner \psi: \alpha \in \Lambda_{7}^{1}\right\} \\
\Lambda_{27}^{3} & =\left\{\chi \in \Lambda^{3}: \chi_{a b c}=h_{[a}^{d} \varphi_{b c] d} \text { for } h_{a b} \text { traceless, symmetric }\right\} \\
\Lambda_{1}^{4} & =\left\{f \psi: f \in C^{\infty}(M)\right\} \\
\Lambda_{7}^{4} & =\left\{\alpha \wedge \varphi: \alpha \in \Lambda_{7}^{1}\right\} \\
\Lambda_{27}^{4} & =\left\{\chi \in \Lambda^{4}: \chi_{a b c d}=h_{[a}^{e} \psi_{b c d] e} \text { for } h_{a b} \text { traceless, symmetric }\right\} \\
\Lambda_{7}^{5} & =\left\{\alpha \wedge \psi: \alpha \in \Lambda_{7}^{1}\right\} \\
\Lambda_{14}^{5} & =\left\{\omega \wedge \varphi: \omega \in \Lambda_{14}^{2}\right\} .
\end{aligned}
$$

In particular, we see that the 7 -dimensional component of $\Lambda^{3}$ is defined by 1 -forms (or equivalently, vectors), and the 27-dimensional component is given by traceless symmetric tensors. For convenience, and following [3], we will adopt the following notation for the map from symmetric tensors into $\Lambda^{3}$ :

$$
\mathrm{i}_{\varphi}: \operatorname{Sym}^{2}\left(V^{*}\right) \longrightarrow \Lambda^{3} \text { given by } \mathrm{i}_{\varphi}(h)_{a b c}=h_{[a}^{d} \varphi_{|d| b c]}
$$

and similarly, for the map from symmetric tensors into $\Lambda^{4}$ :

$$
\mathrm{i}_{\psi}: \operatorname{Sym}^{2}\left(V^{*}\right) \longrightarrow \Lambda^{4} \text { given by } \mathrm{i}_{\psi}(h)_{a b c d}=h_{[a}^{e} \psi_{|e| b c d]} .
$$

Note that here the vertical bars around indices mean that those indices are not included in the antisymmetrization.

It is sometimes useful to be able to find projections of given $p$-form onto the different components. Here we collect some of these results [10, 14]:

Proposition 2.6. Suppose $\omega$ is a 2-form. Then the projections $\pi_{7}(\omega)$ and $\pi_{14}(\omega)$ onto $\Lambda_{7}^{2}$ and $\Lambda_{14}^{2}$, respectively, are given by

$$
\begin{aligned}
\pi_{7}(\omega) & \left.=\alpha\lrcorner \varphi \quad \text { where } \alpha=\frac{1}{6} \omega\right\lrcorner \varphi \\
\pi_{14}(\omega) & \left.=\frac{2}{3} \omega-\frac{1}{6} \omega\right\lrcorner \psi .
\end{aligned}
$$

Similarly, if $\eta$ is a 5 -form, the projections $\pi_{7}(\eta)$ and $\pi_{14}(\eta)$ onto $\Lambda_{7}^{5}$ and $\Lambda_{14}^{5}$, respectively, are given by

$$
\begin{aligned}
\pi_{7}(\eta) & \left.=\alpha \wedge \psi \quad \text { where } \alpha=\frac{1}{72} \psi\right\lrcorner \eta \\
\pi_{14}(\eta) & \left.\left.\left.=\omega \wedge \varphi \text { where } \omega=\frac{1}{9} \varphi\right\lrcorner \eta-\frac{1}{36}(\varphi\lrcorner \eta\right)\right\lrcorner \psi .
\end{aligned}
$$

Proof. The proof of (2.13). is given in [14]. For the decomposition of 5-forms, consider

$$
\eta=\alpha \wedge \psi+\omega \wedge \varphi
$$


where $\alpha$ is a 1 -form and $\omega \in \Lambda_{14}^{2}$. Then,

$$
(\psi\lrcorner \eta)_{m}=5 \psi^{a b c d} \alpha_{[a} \psi_{b c d] m}+10 \psi^{a b c d} \omega_{[a b} \varphi_{c d] m} .
$$

Using the contractions between $\varphi$ and $\psi$ from Proposition 2.4, we find that

$$
\begin{aligned}
(\psi\lrcorner \eta)_{m} & =72 \alpha_{m}+24 \varphi_{m}{ }^{a b} \omega_{a b} \\
& =72 \alpha_{m}
\end{aligned}
$$

since $\omega\lrcorner \varphi=0$ for $\omega \in \Lambda_{14}^{2}$. Hence we get the $\pi_{7}$ projection.

Now for $\eta$ as in (2.15), from definition of the Hodge star, we have,

$$
\begin{aligned}
(\varphi\lrcorner \eta)_{a b} & =\frac{1}{2} \varepsilon_{a b c d e}^{m n}(* \eta)_{m n} \varphi^{a b c} \\
& =3(* \eta\lrcorner \psi)_{a b} \\
& =12\left(\pi_{7} * \eta\right)_{a b}-6\left(\pi_{14} * \eta\right)_{a b} .
\end{aligned}
$$

In particular, from (2.13),

$$
\left.\left.\left.\left.\pi_{14}(\varphi\lrcorner \eta\right)=\frac{2}{3}(\varphi\lrcorner \eta\right)-\frac{1}{6}(\varphi\lrcorner \eta\right)\right\lrcorner \psi
$$

and so,

$$
\left.\left.\left.\pi_{14}(* \eta)=-\frac{1}{9}(\varphi\lrcorner \eta\right)+\frac{1}{36}(\varphi\lrcorner \eta\right)\right\lrcorner \psi .
$$

However,

$$
\begin{aligned}
*\left(\pi_{14}(* \eta) \wedge \varphi\right)_{m n} & =\frac{1}{12} \varepsilon_{m n}{ }^{a b c d e} \pi_{14}(* \eta)_{a b} \varphi_{c d e} \\
& \left.=\frac{1}{2}\left(\pi_{14}(* \eta)\right\lrcorner \psi\right)_{m n} \\
& =-\pi_{14}(* \eta)_{m n} .
\end{aligned}
$$

Hence,

$$
\pi_{14}(\eta)=-\pi_{14}(* \eta) \wedge \varphi
$$

Proposition 2.7. Suppose $\chi$ is a 3 -form. Then the projections $\pi_{1}(\chi), \pi_{7}(\chi)$ and $\pi_{27}(\chi)$ onto $\Lambda_{1}^{3}, \Lambda_{7}^{3}$ and $\Lambda_{27}^{3}$, respectively, are given by

$$
\begin{aligned}
& \left.\pi_{1}(\chi)=a \varphi \text { where } a=\frac{1}{42} \chi\right\lrcorner \varphi \\
& \left.\left.\pi_{7}(\chi)=\omega\right\lrcorner \psi \text { where } \omega=-\frac{1}{24} \chi\right\lrcorner \psi \\
& \left.\pi_{27}(\chi)=\mathrm{i}_{\varphi}(h) \text { where } h_{a b}=\frac{3}{4} \chi_{m n(a} \varphi_{b)}^{m n}-\frac{3}{28}(\chi\lrcorner \varphi\right) g_{a b} \text {. }
\end{aligned}
$$

Similarly, if $\chi$ is a 4-form, the projections are

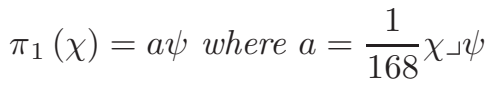

$$
\begin{aligned}
& \left.\pi_{7}(\chi)=\omega \wedge \varphi \text { where } \omega=-\frac{1}{24} \varphi\right\lrcorner \chi \\
& \left.\pi_{27}(\chi)=\mathrm{i}_{\psi}(h) \text { where } h_{a b}=-\frac{1}{3} \chi_{m n p(a} \psi_{b)}^{m n p}-\frac{1}{21}(\chi\lrcorner \psi\right) g_{a b} \text {. }
\end{aligned}
$$

Proof. The proof of (2.20) can be found in either [10] or [14]. 
3. $G_{2}$-structure torsion. As before, suppose $M$ is a 7-dimensional manifold with a $G_{2}$-structure $(\varphi, g)$. The metric $g$ defines a reduction of the frame bundle to a principal $S O(7)$-subbundle $Q$, that is, a subbundle of oriented orthonormal frames. The metric also defines a Levi-Civita connection $\nabla$ on the tangent bundle $T M$, and hence on $F$. However, the $G_{2}$-invariant 3 -form $\varphi$ reduces the orthonormal bundle further to a principal $G_{2}$-subbundle $P$. We can then restrict the Levi-Civita connection to $P$. On $P$ we can uniquely decompose $\nabla$ as

$$
\nabla=\bar{\nabla}+\mathcal{T}
$$

where $\bar{\nabla}$ is a $G_{2}$-compatible canonical connection $\bar{\nabla}$ on $P$, taking values in $\mathfrak{g}_{2} \subset \mathfrak{s o}(7)$, while $\mathcal{T}$ is a 1 -form taking values in $\mathfrak{g}_{2}^{\perp} \subset \mathfrak{s o}(7)$. This 1 -form $\mathcal{T}$ is known as the intrinsic torsion of the $G_{2}$-structure. The intrinsic torsion is precisely the obstruction to the Levi-Civita connection being $G_{2}$-compatible. Note that $\mathfrak{s o}(7)$ splits according to $G_{2}$ representations as

$$
\mathfrak{s o}(7) \cong \Lambda^{2} V \cong \Lambda_{7}^{2} \oplus \Lambda_{14}^{2}
$$

but $\Lambda_{14}^{2} \cong \mathfrak{g}_{2}$, so the complement $\mathfrak{g}_{2}^{\perp} \cong \Lambda_{7}^{2} \cong V$. Hence $\mathcal{T}$ can be represented by a tensor $T_{a b}$ which lies in $W \cong V \otimes V$. Now, since $\varphi$ is $G_{2}$-invariant, it is $\bar{\nabla}$-parallel, so the torsion is determined by $\nabla \varphi$.

Following [14], consider the 3 -form $\nabla_{X} \varphi$ for some vector field $X$. It is easy to see

$$
\nabla_{X} \varphi \in \Lambda_{7}^{3}
$$

and thus overall,

$$
\nabla \varphi \in \Lambda_{7}^{1} \otimes \Lambda_{7}^{3} \cong W
$$

Thus $\nabla \varphi$ lies in the same space as $T_{a b}$ and thus completely determines it. Given (3.3), we can write

$$
\nabla_{a} \varphi_{b c d}=T_{a}{ }^{e} \psi_{e b c d}
$$

where $T_{a b}$ is the full torsion tensor. From this we can also write

$$
T_{a}{ }^{m}=\frac{1}{24}\left(\nabla_{a} \varphi_{b c d}\right) \psi^{m b c d} .
$$

This 2-tensor fully defines $\nabla \varphi$ since pointwise, it has 49 components and the space $W$ is also 49-dimensional (pointwise). In general we can split $T_{a b}$ into torsion components as

$$
\left.T=\tau_{1} g+\tau_{7}\right\lrcorner \varphi+\tau_{14}+\tau_{27}
$$

where $\tau_{1}$ is a function, and gives the $\mathbf{1}$ component of $T$. We also have $\tau_{7}$, which is a 1-form and hence gives the $\mathbf{7}$ component, and, $\tau_{14} \in \Lambda_{14}^{2}$ gives the $\mathbf{1 4}$ component and $\tau_{27}$ is traceless symmetric, giving the $\mathbf{2 7}$ component. Note that the normalization of these components is different from [14]. Hence we can split $W$ as

$$
W=W_{1} \oplus W_{7} \oplus W_{14} \oplus W_{27} .
$$

Originally the torsion of $G_{2}$-structures was studied by Fernández and Gray [6], and their analysis revealed that there are in fact a total of 16 torsion classes of $G_{2^{-}}$ structures. Later on, Karigiannis rederived the splitting (3.7) using simpler computational arguments [14]. The 16 torsion classes arise as the subsets of $W$ which $\nabla \varphi$ belongs to. 
Note that our notation differs from Fernández and Gray. Our $\tau_{1}$ corresponds to their $\tau_{0}, \tau_{7}$ corresponds to $\tau_{4}, \tau_{14}$ corresponds to $\tau_{2}$ and $\tau_{27}$ corresponds to $\tau_{3}$.

Moreover, as shown in [14], the torsion components $\tau_{i}$ relate directly to the expression for $d \varphi$ and $d \psi$. In fact, in our notation,

$$
\begin{aligned}
& d \varphi=4 \tau_{1} \psi-3 \tau_{7} \wedge \varphi-3 * i_{\varphi}\left(\tau_{27}\right) \\
& d \psi=-4 \tau_{7} \wedge \psi-2 * \tau_{14} .
\end{aligned}
$$

Similarly to (3.4), we can express the covariant derivative of $\psi$ in terms of $T$.

Lemma 3.1. Given a $G_{2}$-structure defined by 3 -form $\varphi$, with torsion $T_{a}{ }^{m}$ given by (3.5), the covariant derivative of the corresponding 4-form $\psi$ is given by

$$
\nabla_{a} \psi_{b c d e}=-4 T_{a[b} \varphi_{c d e]} .
$$

Proof. Consider the identity (2.9a):

$$
\varphi_{a b c} \varphi_{m n}^{c}=g_{a m} g_{b n}-g_{a n} g_{b m}+\psi_{a b m n} .
$$

Applying the covariant derivative to both sides, we get

$$
\nabla_{e} \psi_{a b m n}=\left(\nabla_{e} \varphi_{a b c}\right) \varphi_{m n}^{c}+\varphi_{a b c}\left(\nabla_{e} \varphi_{m n}^{c}\right) .
$$

Now using (3.4) and using contraction identities between $\varphi$ and $\psi$, we get (3.9).

Suppose $d \varphi=d \psi=0$. Then this means that all four torsion components vanish and hence $T=0$, and as a consequence $\nabla \varphi=0$. The converse is trivially true. This result is originally due to Fernández and Gray [6]. Moreover, a $G_{2}$-structure is torsion-free if and only if the holonomy of the corresponding metric is contained in $G_{2}[12]$.

The torsion tensor $T_{a b}$ and hence the individual components $\tau_{1}, \tau_{7}, \tau_{14}$ and $\tau_{27}$ must also satisfy certain differential conditions. For the exterior derivative $d, d^{2}=0$, so from (3.8), must have

$$
\begin{aligned}
d\left(4 \tau_{1} \psi-3 \tau_{7} \wedge \varphi-3 * i_{\varphi}\left(\tau_{27}\right)\right) & =0 \\
d\left(4 \tau_{7} \wedge \psi+2 * \tau_{14}\right) & =0 .
\end{aligned}
$$

Alternatively, note that we have

$$
\begin{aligned}
\left(d^{2} \varphi\right)_{a b c d e} & =20 \nabla_{[a} \nabla_{b} \varphi_{c d e]} \\
& =20 \nabla_{[a} T_{b}^{f} \psi_{|f| c d e]}
\end{aligned}
$$

and

$$
\begin{aligned}
\left(d^{2} \psi\right)_{a b c d e f} & =30 \nabla_{[a} \nabla_{b} \psi_{c d e f]} \\
& =30 \nabla_{[a} T_{b c} \varphi_{d e f]} .
\end{aligned}
$$

So in particular, we get conditions

$$
\begin{aligned}
\nabla_{[a} T_{b}{ }^{f} \psi_{|f| c d e]} & =0 \\
\nabla_{[a} T_{b c} \varphi_{\text {def }]} & =0 .
\end{aligned}
$$

From these, we get the following conditions.

Proposition 3.2. The torsion tensor $T_{a b}$ of a $G_{2}$-structure $\varphi$ satisfies the following consistency conditions 
1 .

$$
\varphi^{a b c} T_{b c} T_{a m}-T^{b d} T_{b}^{c} \varphi_{m d c}-\psi_{m}{ }^{a b c} \nabla_{a} T_{b c}-(\operatorname{Tr} T) \varphi_{m}{ }^{a b} T_{a b}=0
$$

2.

$$
\nabla_{m}(\operatorname{Tr} T)-\nabla_{a} T_{m}^{a}-T_{m c} \varphi^{a b c} T_{a b}=0
$$

3.

$$
\begin{aligned}
0= & -\varphi_{m n}{ }^{c} \nabla_{c}(\operatorname{Tr} T)+6 T_{a[m} \psi_{n]}^{a b c} T_{b c}+2\left(\nabla_{a} T_{[m|b|}\right) \varphi_{n]}^{a b} \\
& +2\left(\nabla_{[m} T_{|a b|}\right) \varphi_{n]}^{a b}+2 \psi_{m n a b} T^{c a} T_{b c}+2(\operatorname{Tr} T) \psi_{m n}{ }^{a b} T_{a b}+ \\
& +\varphi_{m n a} \varphi_{b c d} T^{c d} T^{b a}-2 \varphi_{m n a} \varphi_{b c d} T^{c d} T^{a b}+2 T_{[m}{ }^{a} T_{|a| n]}- \\
& 4 \varphi_{[m}^{a b} \nabla_{\mid a} T_{b \mid n]}-2(\operatorname{Tr} T) T_{[m n]}+\varphi_{m n}{ }^{a} \nabla_{b} T_{a}{ }^{b}
\end{aligned}
$$

Proof. Let us first look at $\left(d^{2} \varphi\right)_{a b c d e}=20 \nabla_{[a} T_{b}{ }^{f} \psi_{|f| c d e]}$. We have

$$
\begin{aligned}
\nabla_{a} T_{b}{ }^{f} \psi_{f c d e} & =\left(\nabla_{a} T_{b}{ }^{f}\right) \psi_{f c d e}+T_{b}{ }^{f}\left(\nabla_{a} \psi_{f c d e}\right) \\
& =\left(\nabla_{a} T_{b}{ }^{f}\right) \psi_{f c d e}-4 T_{b}{ }^{f} T_{[a f} \varphi_{c d e]} \\
& =\left(\nabla_{a} T_{b}{ }^{f}\right) \psi_{f c d e}-\frac{4}{5} T_{b}{ }^{f} T_{[a|f|} \varphi_{c d e]}+\frac{4}{5} T_{b}{ }^{f} T_{f[a} \varphi_{c d e]}+\frac{12}{5} T_{b}{ }^{f} T_{[a c} \varphi_{d e] f} .
\end{aligned}
$$

In the last line we have used the fact that when $T_{[a f} \varphi_{c d e]}$ is expanded, out of the twenty distinct terms, twelve have the index $f$ on $\varphi$, four terms have $f$ on the first index of $T$ and four have $f$ on the second index of $T$. Now, anti-symmetrizing on $a$, $b, c, d$ and $e$, we obtain

$$
\nabla_{[a} T_{b}^{f} \psi_{|f| c d e]}=\left(\nabla_{[a} T_{b}^{f}\right) \psi_{|f| c d e]}+\frac{4}{5} T_{[a}^{f} T_{b|f|} \varphi_{c d e]}-\frac{4}{5} T_{[a}^{f} T_{|f| b} \varphi_{c d e]}-\frac{12}{5} T_{[a}^{f} T_{b c} \varphi_{d e] f} .
$$

Using Proposition 2.6, we find the projections of (3.14) onto $\Lambda_{14}^{5}$ and $\Lambda_{7}^{5}$. Considering the corresponding 2-form in $\Lambda_{14}^{2}$ we obtain (3.13). From the $\Lambda_{7}^{5}$ component, we get

$$
\begin{aligned}
0= & 2 \nabla_{m}(\operatorname{Tr} T)-2 \nabla_{a} T_{m}{ }^{a}-2 T_{m c} \varphi^{a b c} T_{a b}+\varphi^{a b c} T_{b c} T_{a m} \\
& -T^{b d} T_{b}^{c} \varphi_{m d c}-\psi_{m}{ }^{a b c} \nabla_{a} T_{b c}-(\operatorname{Tr} T) \varphi_{m}{ }^{a b} T_{a b} .
\end{aligned}
$$

Let us now look at $\left(d^{2} \psi\right)_{a b c d e f}=30 \nabla_{[a} T_{b c} \varphi_{d e f]}$. This is now a 6 -form, so taking the Hodge star we get 1 -form and hence automatically another 7 component. From this we immediately obtain

$$
\varphi^{a b c} T_{b c} T_{a m}-T^{b d} T_{b}^{c} \varphi_{m d c}-\psi_{m}^{a b c} \nabla_{a} T_{b c}-(\operatorname{Tr} T) \varphi_{m}^{a b} T_{a b}=0,
$$

that is, (3.11). Subtracting this condition from (3.15), we obtain (3.12).

Alternatively, proposition 3.2 can also be obtained from the "Bianchi-type identity" for the torsion, which was obtained in [14] from diffeomorphism invariance. In our sign convention this identity is

$$
\mathcal{T}_{a b c}:=\nabla_{a} T_{b c}-\nabla_{b} T_{a c}+T_{a m} T_{b n} \varphi_{c}^{m n}-\frac{1}{2} R_{a b m n} \varphi_{c}^{m n}=0 .
$$


The quantity $\mathcal{T}_{a b c}$ is then pointwise in the $\mathbf{7} \otimes(\mathbf{7} \oplus \mathbf{1 4})$ representation of $G_{2}$, which, as shown in [5], decomposes as

$$
\mathbf{7} \otimes(\mathbf{7} \oplus \mathbf{1 4}) \cong(\mathbf{1} \oplus \mathbf{7} \oplus \mathbf{1 4} \oplus \mathbf{2 7}) \oplus(\mathbf{7} \oplus \mathbf{2 7} \oplus \mathbf{6 4}) .
$$

We can consider different projection of $\mathcal{T}$. In particular, let

$$
\begin{gathered}
\left(\mathcal{T}_{1}\right)_{a b}=\mathcal{T}_{a c d} \varphi^{c d} \\
\left(\mathcal{T}_{2}\right)_{a b}=\mathcal{T}_{c d a} \varphi^{c d}{ }_{b} \\
R_{a b}=\left(\nabla_{a} T_{n m}-\nabla_{n} T_{a m}\right) \varphi_{b}^{n m}-T_{a n} T_{b}^{n}+\operatorname{Tr}(T) T_{a b} \\
+T_{a c} T_{n m} \psi_{b}^{n m c} \\
R_{m n p q} \varphi_{a}^{m n} \varphi_{b}^{p q}=4\left(\nabla_{m} T_{n a}\right) \varphi_{b}^{m n}+2 T_{p m} T_{q n} \varphi_{a}^{m n} \varphi_{b}^{p q} .
\end{gathered}
$$

Then, from (3.16), $\mathcal{T}_{1}=0$ and $\mathcal{T}_{2}=0$. These conditions then giveThe expression (3.18) for the Ricci curvature in terms of the torsion has previously been derived by Bryant in [3] and Karigiannis in [14]. Our expression differs from [14] due different sign convention for $\psi$. This also leads to a different sign for $T_{a b}$.

Taking the trace of both (3.18) and (3.19) gives the same expression for the scalar curvature, and thus gives the $\mathbf{1}$ component of $\mathcal{T}$. The traceless symmetric parts then give the two distinct $\mathbf{2 7}$ components of $\mathcal{T}$. The left hand sides of (3.20) are both symmetric, so the antisymmetric parts of (3.20) must also vanish. Taking the 7 components of both (3.18) and (3.19) give the two 7 components of $\mathcal{T}$ which are, in fact, distinct linear combinations of (3.11) and (3.12). It can then also be shown that the 14 components of (3.11) and (3.12) are actually equal. This then gives the unique 14 component of $\mathcal{T}$, which is actually also equal to (3.13).

Since we are interested in particular torsion classes, which are given by torsion components, it is helpful to have conditions corresponding to (3.11)-(3.13) in terms of individual torsion components $\tau_{1}, \tau_{7}, \tau_{14}$ and $\tau_{27}$.

Proposition 3.3. Given the decomposition (3.6) of the full torsion tensor $T_{a b}$ into components $\tau_{1}, \tau_{7}, \tau_{14}$ and $\tau_{27}$, these components satisfy the following consistency conditions:

1.

$$
\nabla_{a}\left(\tau_{14}\right)_{m}^{a}+2 \varphi_{m}^{a b} \nabla_{a}\left(\tau_{7}\right)_{b}+4\left(\tau_{7}\right)_{a}\left(\tau_{14}\right)_{m}^{a}=0
$$

2.

$$
\nabla_{m} \tau_{1}-\frac{1}{2} \varphi_{m}^{a b} \nabla_{a}\left(\tau_{7}\right)_{b}-\frac{1}{6} \nabla_{a}\left(\tau_{27}\right)_{m}^{a}-\left(\tau_{7}\right)_{a}\left(\tau_{27}\right)_{m}^{a}-\left(\tau_{7}\right)_{m} \tau_{1}=0
$$

3.

$$
\begin{aligned}
0= & \varphi_{m n a} \nabla_{b}\left(\tau_{27}\right)^{a b}+6 \nabla_{a}\left(\tau_{27}\right)_{b[m} \varphi_{n]}^{a b}-24 \tau_{1}\left(\tau_{14}\right)_{m n} \\
& -18\left(\frac{2}{3} \nabla_{[m}\left(\tau_{7}\right)_{n]}-\frac{1}{6} \psi_{m n}^{a b} \nabla_{a}\left(\tau_{7}\right)_{b}\right) \\
& -18\left(\frac{2}{3}\left(\tau_{14}\right)_{a[m}\left(\tau_{27}\right)_{n]}^{a}-\frac{1}{6} \psi_{m n}^{a b}\left(\tau_{14}\right)_{a}^{c}\left(\tau_{27}\right)_{b c}\right)
\end{aligned}
$$


For some of the torsion classes, these conditions simplify, which we summarize below.

\begin{tabular}{|c|c|c|}
\hline Torsion class & Condition & In coordinates \\
\hline$W_{1}$ & $d \tau_{1}=0$ & $\nabla_{m} \tau_{1}=0$ \\
\hline$W_{7}$ & $d \tau_{7}=0$ & $\nabla_{[m}\left(\tau_{7}\right)_{n]}=0$ \\
\hline$W_{14}$ & $d^{*} \tau_{14}=0$ & $\nabla_{a}\left(\tau_{14}\right)^{a}{ }_{m}=0$ \\
\hline$W_{27}$ & $d^{*} i_{3}\left(\tau_{27}\right)=0$ & $\begin{array}{l}\nabla_{a}\left(\tau_{27}\right)^{a}{ }_{m}=0 \\
\nabla_{a}\left(\tau_{27}\right)_{b[m} \varphi_{n]}^{a b}=0\end{array}$ \\
\hline$W_{1} \oplus W_{7}$ & $\begin{array}{l}\tau_{7}=d\left(\log \left|\tau_{1}\right|\right) \text { if } \tau_{1} \text { nowhere zero } \\
d \tau_{7}=0 \text { and } \tau_{1}=0 \text { otherwise }\end{array}$ & \\
\hline$W_{1} \oplus W_{14}$ & $\tau_{1}=0 \quad$ or $\tau_{14}=0$ & \\
\hline$W_{1} \oplus W_{27}$ & $\left.d^{*} i_{3}\left(\tau_{27}\right)=-\frac{4}{3}\left(d \tau_{1}\right)\right\lrcorner \varphi$ & $\begin{array}{l}\nabla_{a}\left(\tau_{27}\right)_{m}^{a}=6 \nabla_{m} \tau_{1} \\
\nabla_{a}\left(\tau_{27}\right)_{b[m} \varphi_{n]}{ }^{a b} \\
\quad+\frac{1}{6} \varphi_{m n a} \nabla_{b}\left(\tau_{27}\right)^{a b}=0\end{array}$ \\
\hline$W_{7} \oplus W_{14}$ & $\begin{array}{l}d \tau_{7}=0 \\
\left.d^{*} \tau_{14}=4 \tau_{7}\right\lrcorner \tau_{14}\end{array}$ & $\begin{array}{l}\nabla_{[m}\left(\tau_{7}\right)_{n]}=0 \\
\nabla_{a}\left(\tau_{14}\right)^{a}{ }_{m} \\
\quad+4\left(\tau_{7}\right)_{a}\left(\tau_{14}\right)^{a}{ }_{m}=0\end{array}$ \\
\hline
\end{tabular}

To obtain these conditions, we simply use the expressions (3.21) to (3.23), and set the relevant torsion components to zero. For the class $W_{1} \oplus W_{7}$, the characterization that either $\tau_{7}$ is the gradient of $\log \left|\tau_{1}\right|$ or $\tau_{1}$ is zero everywhere was given by Cleyton and Ivanov in ([4]).

4. Deformations of $G_{2}$-structures. Suppose we have a $G_{2}$-structure on $M$ defined by the 3 -form $\varphi$. In $[10,9]$ we considered small deformations of $G_{2}$-structures and then expanded related quantities such as the metric $g$, the volume form $\sqrt{\operatorname{det} g}$ and the 4 -form $\psi$ up to a certain order in the small parameter. We will now deduce some results about more general deformations. Suppose we have a deformation for some 3 -form $\chi$

$$
\varphi \longrightarrow \tilde{\varphi}=\varphi+\chi
$$

In $[10,9]$ it was pointed out that generically it is difficult to obtain a closed form expression for $\tilde{g}$ and $\tilde{\psi}$. One of the challenges was to obtain a closed form expression for $\operatorname{det} \tilde{g}$. However it turns out that there is an easy way to do this, even if obtaining the full explicit expression is still computationally challenging. Note that we will use upper indices with tilde to denote indices raised with the deformed metric $\tilde{g}$.

Lemma 4.1. Given a deformation of $\varphi$ as in (4.1), the related quantities $\tilde{g}, \tilde{\psi}$ and $\operatorname{det} g$ are given by:

$$
\begin{aligned}
\tilde{g}_{a b} & =\left(\frac{\operatorname{det} g}{\operatorname{det} \tilde{g}}\right)^{\frac{1}{2}} s_{a b} \\
\tilde{\psi}_{a b c d} & =\left(\frac{\operatorname{det} g}{\operatorname{det} \tilde{g}}\right)^{\frac{5}{2}}\left(\psi^{m n p q}+* \chi^{m n p q}\right) s_{m a} s_{n b} s_{p c} s_{q d}
\end{aligned}
$$

where

$$
s_{a b}=g_{a b}+\frac{1}{2} \chi_{m n(a} \varphi_{b)}^{m n}+\frac{1}{8} \chi_{a m n} \chi_{b p q} \psi^{m n p q}+\frac{1}{24} \chi_{a m n} \chi_{b p q}(* \chi)^{m n p q} .
$$


Moreover,

$$
\tilde{\psi}^{\tilde{a} \tilde{b} \tilde{c} \tilde{d}}=\tilde{g}^{a m} \tilde{g}^{b n} \tilde{g}^{c p} \tilde{g}^{d q} \tilde{\psi}_{m n p q}=\left(\frac{\operatorname{det} g}{\operatorname{det} \tilde{g}}\right)^{\frac{1}{2}}\left(\psi^{m n p q}+* \chi^{m n p q}\right) .
$$

Proof. From [10] we know the expression (4.2a) with $s_{a b}$ as in (4.3). Now,

$$
\begin{aligned}
\tilde{\psi}_{a b c d} & =\tilde{*}(\varphi+\chi)_{a b c d} \\
& =\frac{1}{3 !} \frac{1}{\sqrt{\operatorname{det} \tilde{g}}} \hat{\varepsilon}^{m n p q r s t}\left(\varphi_{r s t}+\chi_{r s t}\right) \tilde{g}_{m a} \tilde{g}_{n b} \tilde{g}_{p c} \tilde{g}_{q d} .
\end{aligned}
$$

Here $\tilde{\varepsilon}^{a b c d r s t}$ refers to the alternating symbol which takes values 0 and \pm 1 . Hence, using (4.2a), we get

$$
\begin{aligned}
\tilde{\psi}_{a b c d} & =\left(\frac{\operatorname{det} g}{\operatorname{det} \tilde{g}}\right)^{\frac{1}{2}}\left(\psi^{m n p q}+* \chi^{m n p q}\right) \tilde{g}_{m a} \tilde{g}_{n b} \tilde{g}_{p c} \tilde{g}_{q d} \\
& =\left(\frac{\operatorname{det} g}{\operatorname{det} \tilde{g}}\right)^{\frac{5}{2}}\left(\psi^{m n p q}+* \chi^{m n p q}\right) s_{m a} s_{n b} s_{p c} s_{q d}
\end{aligned}
$$

which is precisely $(4.2 \mathrm{~b})$. Incidentally, by raising indices in (4.5) using $\tilde{g}$, we obtain (4.4).

Lemma 4.1 thus shows that both the deformed metric $\tilde{g}$ and the 4 -form $\tilde{\psi}$ are expressible as polynomials in $\chi$ multiplied by an integer power of the ratio of the volume forms $\left(\frac{\operatorname{det} g}{\operatorname{det} \tilde{g}}\right)^{\frac{1}{2}}$. Therefore, it is important be able to evaluate this ratio.

To obtain more identities we can use contraction formulae for $\tilde{\varphi}$ and $\tilde{\psi}$. Since $\tilde{\varphi}$ defines a $G_{2}$-structure, $\tilde{\varphi}$ and $\tilde{\psi}$ satisfy the same identities as $\varphi$ and $\psi$ in Proposition 2.4. So, in particular, we have

$$
\begin{aligned}
\tilde{\varphi}_{a}^{\tilde{b} \tilde{c}} & =\frac{1}{4} \tilde{\varphi}_{a m n} \tilde{\psi}^{\tilde{m} \tilde{n} \tilde{b} \tilde{c}}=\frac{1}{4}\left(\frac{\operatorname{det} g}{\operatorname{det} \tilde{g}}\right)^{\frac{1}{2}}\left(\varphi_{a m n}+\chi_{a m n}\right)\left(\psi^{m n b c}+* \chi^{m n b c}\right) \\
& =\frac{1}{4}\left(\frac{\operatorname{det} g}{\operatorname{det} \tilde{g}}\right)^{\frac{1}{2}}\left(4 \varphi_{a}^{b c}+\varphi_{a m n} * \chi^{m n b c}+\chi_{a m n} \psi^{m n b c}+\chi_{a m n} * \chi^{m n b c}\right) .
\end{aligned}
$$

This expression is very simple from computational point of view, since it does not involve the quantity $s_{a b}$ (apart from the determinant factor).

We can then use (4.6) together with other contraction identities to get closed expressions for the inverse metric $\tilde{g}^{\tilde{a} \tilde{b}}$ and the ratio of volume forms $\left(\frac{\operatorname{det} g}{\operatorname{det} \tilde{g}}\right)^{\frac{1}{2}}$.

Proposition 4.2. Given a deformation of $\varphi$ as in (4.1), and the corresponding deformation of the metric (4.2a), the deformed inverse metric is given by

$$
\tilde{g}^{\tilde{a} \tilde{m}}=\left(\frac{\operatorname{det} g}{\operatorname{det} \tilde{g}}\right) \gamma^{a m}
$$

where

$$
\begin{aligned}
\gamma^{a m}= & g^{a m}-\frac{1}{96} * \chi_{b c d}^{a} * \chi_{p q r}^{m} \varphi^{b c p} \varphi^{d q r}-\frac{1}{48} * \chi_{b c d}^{(a} * \chi_{p q r}^{m)} \varphi^{b c p} \chi^{d q r} \\
& -\frac{1}{48} * \chi_{b c d}^{(a} \psi_{p q r}^{m)} \chi^{b c p} \chi^{d q r}-\frac{1}{96} \psi_{b c d}^{a} \psi_{p q r}^{m} \chi^{b c p} \chi^{d q r}-\frac{1}{96} * \chi_{b c d}^{a} * \chi_{p q r}^{m} \chi^{b c p} \chi^{d q r} \\
& -\frac{1}{4} \chi_{b c}^{(a} \varphi^{m) b c}+\frac{1}{6} * \chi_{b c d}^{(a} \varphi^{m) b}{ }_{e}^{c d e}+\frac{1}{12} * \chi_{b c d}^{(a} \psi^{m) b c d}+\frac{1}{12} g^{a m} \chi^{b c d} \varphi_{b c d}
\end{aligned}
$$


and

$$
\left(\frac{\operatorname{det} \tilde{g}}{\operatorname{det} g}\right)^{\frac{3}{2}}=\frac{1}{7} \gamma^{a m} s_{a m}
$$

for $s_{a m}$ as in (4.3).

Proof. We have the following $G_{2}$-structure contraction identity for $\tilde{\varphi}$ :

$$
\tilde{\varphi}_{a b c} \tilde{\varphi}_{m}{ }^{\tilde{b} \tilde{c}}=6 \tilde{g}_{a m}
$$

Hence,

$$
\tilde{\varphi}_{c}^{\tilde{a} \tilde{b}} \tilde{\varphi}_{b}^{\tilde{m} \tilde{c}}=-6 \tilde{g}^{\tilde{a} \tilde{m}}
$$

From (4.6), we thus have

$$
\begin{aligned}
\tilde{g}^{\tilde{a} \tilde{m}}= & -\frac{1}{6} \tilde{\varphi}_{c}^{\tilde{a} \tilde{b}} \tilde{\varphi}_{b}^{\tilde{m} \tilde{c}} \\
= & -\frac{1}{96}\left(\frac{\operatorname{det} g}{\operatorname{det} \tilde{g}}\right)\left(4 \varphi_{c}^{a b}+\varphi_{c d e} * \chi^{\text {deab }}+\chi_{c d e} \psi^{\text {deab }}+\chi_{c d e} * \chi^{\text {deab }}\right) \times \\
& \left(4 \varphi_{b}^{m c}+\varphi_{b p q} * \chi^{p q m c}+\chi_{b p q} \psi^{p q m c}+\chi_{b p q} * \chi^{p q m c}\right) .
\end{aligned}
$$

Expanding this, and simplifying using $G_{2}$ contraction identities, we get (4.7). Now note that

$$
\tilde{g}^{\tilde{a} \tilde{m}} \tilde{g}_{a m}=\left(\frac{\operatorname{det} g}{\operatorname{det} \tilde{g}}\right)^{\frac{1}{2}} \tilde{g}^{\tilde{a} \tilde{m}} s_{a m}=7
$$

with $s_{a m}$ given by (4.3). Hence,

$$
\left(\frac{\operatorname{det} \tilde{g}}{\operatorname{det} g}\right)^{\frac{3}{2}}=\frac{1}{7} \gamma^{a m} s_{a m}
$$

From Proposition 4.2 we see that $\left(\frac{\operatorname{det} \tilde{g}}{\operatorname{det} g}\right)^{\frac{3}{2}}$ is a polynomial of degree 7 in $\chi$. Therefore, the ratio of volume forms $\left(\frac{\operatorname{det} g}{\operatorname{det} \tilde{g}}\right)^{\frac{1}{2}}$ that appears in Lemma 4.1 is a polynomial of degree 7 in $\chi$ raised to the power $-\frac{1}{3}$. The simplest deformation would be one where $\chi$ lies in $\Lambda_{1}^{3}$, and is hence proportional to $\varphi$. So suppose

$$
\chi=\left(f^{3}-1\right) \varphi
$$

so that

$$
\tilde{\varphi}=f^{3} \varphi .
$$

For convenience, set $A=f^{3}-1$ for now. Then, from (4.3), we get

$$
\begin{aligned}
s_{a b} & =g_{a b}+\frac{1}{2} A \varphi_{m n(a} \varphi_{b)}^{m n}+\frac{1}{8} A^{2} \varphi_{a m n} \varphi_{b p q} \psi^{m n p q}+\frac{1}{24} A^{3} \varphi_{a m n} \varphi_{b p q} \psi^{m n p q} \\
& =\left(1+3 A+3 A^{2}+A^{3}\right) g_{a b} \\
& =(1+A)^{3} g_{a b} \\
& =f^{9} g_{a b}
\end{aligned}
$$


where we have used the contraction identities (2.9). Thus, from (4.2a),

$$
\tilde{g}_{a b}=\left(\frac{\operatorname{det} g}{\operatorname{det} \tilde{g}}\right)^{\frac{1}{2}} f^{9} g_{a b} .
$$

Taking the determinant on both sides, we find that

$$
\operatorname{det} \tilde{g}=f^{14} \operatorname{det} g \text {. }
$$

So in fact,

$$
\begin{aligned}
& \tilde{g}_{a b}=f^{2} g_{a b} \\
& \tilde{g}^{a b}=f^{-2} g^{a b}
\end{aligned}
$$

and so this defines a conformal transformation. We can also then show that

$$
\tilde{\psi}=f^{4} \psi
$$

The next simplest case is when $\chi=v\lrcorner \psi \in \Lambda_{7}^{3}$. Then $* \chi=-v^{b} \wedge \varphi$. From this we can obtain $s_{a b}$ and as it was shown by Karigiannis in [13],

$$
s_{a b}=(1+M) g_{a b}-v_{a} v_{b}
$$

where $M=|v|^{2}$ with the norm taken using $g_{a b}$. Also, using the expressions for $\chi$ and $* \chi$ we can now get $\gamma^{a b}$ from (4.8):

$$
\gamma^{a b}=(1+M)\left(g^{a b}+v^{a} v^{b}\right) .
$$

Substituting this into (4.9), we get:

$$
\left(\frac{\operatorname{det} \tilde{g}}{\operatorname{det} g}\right)^{\frac{3}{2}}=(1+M)^{2}
$$

and therefore,

$$
\begin{aligned}
& \tilde{g}_{a b}=\left(\frac{\operatorname{det} g}{\operatorname{det} \tilde{g}}\right)^{\frac{1}{2}} s_{a b}=(1+M)^{-\frac{2}{3}}\left((1+M) g_{a b}-v_{a} v_{b}\right) \\
& \tilde{g}^{a b}=\left(\frac{\operatorname{det} g}{\operatorname{det} \tilde{g}}\right) \gamma^{a m}=(1+M)^{-\frac{1}{3}}\left(g^{a m}+v^{a} v^{m}\right) .
\end{aligned}
$$

As expected, these are precisely the results obtained in [13]. However the method used here does not depend on the particular form of $s_{a b}$ and hence theoretically is applicable in the case when $\chi \in \Lambda_{27}^{3}$ as well. Also, for later use we will need an expression for $\tilde{\varphi}$ with two raised indices. Using either (4.6) or (4.19b), we see that

$$
\tilde{\varphi}_{a}^{\tilde{b} \tilde{c}}=(1+M)^{-\frac{2}{3}}\left(\varphi_{a}^{b c}-\chi_{a}^{b c}+v^{b} v_{m} \varphi_{a}^{c m}-v^{c} v_{m} \varphi_{a}^{b m}\right) .
$$

Now suppose $\chi \in \Lambda_{27}^{3}$. Then for some traceless, symmetric $h_{a b}$ we can write $\chi$ as

$$
\chi_{a b c}=h_{[a}{ }^{d} \varphi_{b c] d} .
$$


It can then be shown that $* \chi_{a b c d}=-\frac{4}{3} h_{[a}^{e} \psi_{|e| b c d]}$. Now we can substitute both $\chi$ and $* \chi$ into the expression (4.3) for $s_{a b}$, and after some manipulations get

$$
\begin{aligned}
s_{a b}= & g_{a b}+\frac{2}{3} h_{a b}+\frac{2}{9} h_{a}^{c} h_{c b}-\frac{1}{18} \operatorname{Tr}\left(h^{2}\right) g_{a b}-\frac{1}{18} \varphi_{a m n} \varphi_{b p q} h^{m p} h^{n q} \\
& -\frac{1}{27} \varphi_{a m n} \varphi_{b p q} h^{m r} h_{r}^{q} h^{n p}+\frac{1}{81} \operatorname{Tr}\left(h^{3}\right) g_{a b} .
\end{aligned}
$$

This expression for $s_{a b}$ is already rather complicated, and so even getting an explicit closed expression for $\left(\frac{\operatorname{det} \tilde{g}}{\operatorname{det} g}\right)$ becomes a very tough task, which is beyond the scope of this paper. We will instead focus on calculating a general expression for the torsion of the deformed $G_{2}$-structure and will then specialize to the case where $\chi$ lies in $\Lambda_{7}^{3}$.

Now let us consider what happens to the Levi-Civita connection of the deformed metric.

LEMMA 4.3. Given a deformation of $\varphi$ as in (4.1), and the corresponding deformation of the metric (4.2a), the components of the Levi-Civita connection $\tilde{\Gamma}_{a c}^{b}$ corresponding to the new metric $\tilde{g}$ are given by

$$
\tilde{\Gamma}_{a c}^{b}=\Gamma_{a c}^{b}+\delta \Gamma_{a c}^{b}
$$

where

$$
\begin{aligned}
\delta \Gamma_{a c}{ }^{b}= & \frac{1}{2}\left(\frac{\operatorname{det} g}{\operatorname{det} \tilde{g}}\right)^{\frac{1}{2}}\left(\tilde{g}^{\tilde{b} \tilde{d}}\left(\nabla_{c} s_{a d}+\nabla_{a} s_{c d}-\nabla_{d} s_{a c}\right)\right. \\
& \left.-\frac{1}{9}\left(\delta_{a}^{b} \delta_{c}^{e}+\delta_{c}^{b} \delta_{a}^{e}-\tilde{g}_{a c} \tilde{g}^{\tilde{b} \tilde{e}}\right) \tilde{g}^{\tilde{m} \tilde{n}} \nabla_{e} s_{m n}\right)
\end{aligned}
$$

for $s_{a b}$ given by (4.3).

Proof. As it is well known, the components of the Levi-Civita connection are given by

$$
\Gamma_{a c}^{b}=\frac{1}{2} g^{b d}\left(g_{d a, c}+g_{d c, a}-g_{a c, d}\right)
$$

and hence for the modified metric, we have

$$
\tilde{\Gamma}_{a c}^{b}=\frac{1}{2} \tilde{g}^{b d}\left(\tilde{g}_{d a, c}+\tilde{g}_{d c, a}-\tilde{g}_{a c, d}\right) .
$$

To work out the difference between the two connections $\delta \Gamma_{a c}^{b}=\tilde{\Gamma}_{a c}^{b}-\Gamma_{a c}^{b}$, consider the covariant derivative of $\tilde{g}$ with respect to the original connection:

$$
\begin{aligned}
\nabla_{c} \tilde{g}_{a d} & =\tilde{\nabla}_{c} \tilde{g}_{a d}+2 \delta \Gamma_{c}^{e}\left({ }_{a} \tilde{g}_{d) e}\right. \\
& =2 \delta \Gamma_{c(a}^{e} \tilde{g}_{d) e} .
\end{aligned}
$$

It then follows from (4.26) that

$$
\delta \Gamma_{a c}^{b}=\frac{1}{2} \tilde{g}^{b d}\left(\nabla_{c} \tilde{g}_{a d}+\nabla_{a} \tilde{g}_{c d}-\nabla_{d} \tilde{g}_{a c}\right)
$$

We will now work out $\nabla_{c} \tilde{g}_{a d}$ in terms of $s_{a b}$. Let

$$
\mu=\left(\frac{\operatorname{det} \tilde{g}}{\operatorname{det} g}\right)^{\frac{3}{2}} \text {. }
$$


Then, from (4.2a), we have

$$
\begin{aligned}
\nabla_{c} \tilde{g}_{a d} & =\nabla_{c}\left(\mu^{-\frac{1}{3}} s_{a d}\right)=-\frac{1}{3} \mu^{-\frac{4}{3}}\left(\nabla_{c} \mu\right) s_{a d}+\mu^{-\frac{1}{3}} \nabla_{c} s_{a d} \\
& =-\frac{1}{3} \mu^{-1}\left(\nabla_{c} \mu\right) \tilde{g}_{a d}+\mu^{-\frac{1}{3}} \nabla_{c} s_{a d} .
\end{aligned}
$$

Now let us look at $\nabla_{c} \mu$. Using (4.2a) and the definition of the determinant, we get

$$
\mu^{3}=\left(\frac{\operatorname{det} \tilde{g}}{\operatorname{det} g}\right)^{\frac{9}{2}}=\frac{1}{7 !} \frac{1}{\operatorname{det} g} \hat{\varepsilon}^{m n p q r s t} \hat{\varepsilon}^{a b c d e f g} s_{a m} s_{b n} s_{c p} s_{d q} s_{e r} s_{f s} s_{g t},
$$

and similarly,

$$
\tilde{g}^{m u}=\frac{1}{6 !}\left(\frac{\operatorname{det} g}{\operatorname{det} \tilde{g}}\right)^{4} \frac{1}{\operatorname{det} g} \hat{\varepsilon}^{m n p q r s t} \hat{\varepsilon}^{u b c d e f g} s_{b n} s_{c p} s_{d q} s_{e r} s_{f s} s_{g t} .
$$

So, in particular,

$$
\begin{aligned}
\nabla_{c}\left(\mu^{3}\right) & =\nabla_{c}\left(\frac{1}{7 !} \frac{1}{\operatorname{det} g} \hat{\varepsilon}^{m n p q r s t} \hat{\varepsilon}^{a b c d e f g} s_{a m} s_{b n} s_{c p} s_{d q} s_{e r} s_{f s} s_{g t}\right) \\
& =\frac{1}{6 !} \frac{1}{\operatorname{det} g} \hat{\varepsilon}^{m n p q r s t} \hat{\varepsilon}^{a b c d e f g}\left(\nabla_{c} s_{a m}\right) s_{b n} s_{c p} s_{d q} s_{e r} s_{f s} s_{g t} \\
& =\mu^{\frac{8}{3}} \tilde{g}^{m n} \nabla_{c} s_{m n} .
\end{aligned}
$$

Therefore,

$$
\nabla_{c} \mu=\frac{1}{3} \mu^{\frac{2}{3}} \tilde{g}^{m n} \nabla_{c} s_{m n}
$$

and,

$$
\nabla_{c} \tilde{g}_{a d}=-\frac{1}{9} \mu^{-\frac{1}{3}} \tilde{g}_{a d} \tilde{g}^{m n} \nabla_{c} s_{m n}+\mu^{-\frac{1}{3}} \nabla_{c} s_{a d}
$$

Substituting (4.29) into (4.27), we find that

$$
\delta \Gamma_{a c}^{b}=\frac{1}{2} \mu^{-\frac{1}{3}}\left(\tilde{g}^{b d}\left(\nabla_{c} s_{a d}+\nabla_{a} s_{c d}-\nabla_{d} s_{a c}\right)-\frac{1}{9}\left(\delta_{a}^{b} \delta_{c}^{e}+\delta_{c}^{b} \delta_{a}^{e}-\tilde{g}_{a c} \tilde{g}^{b e}\right) \tilde{g}^{m n} \nabla_{e} s_{m n}\right)
$$

and then after substituting $\mu^{-\frac{1}{3}}=\left(\frac{\operatorname{det} g}{\operatorname{det} \tilde{g}}\right)^{\frac{1}{2}}$ we get the result.

REMARK 4.4. For the conformal deformation with $\chi$ given by (4.10), we find that

$$
\delta \Gamma_{a c}^{b}=\frac{1}{9} f^{-9} \partial_{e} f\left(\delta_{c}^{e} \delta_{a}^{b}+\delta_{a}^{e} \delta_{c}^{b}-g^{b e} g_{a c}\right) .
$$

5. Torsion deformations. Suppose we have a deformation of $\varphi$ given by (4.1). Using the results from Sect. 4, we can calculate the deformed torsion.

Lemma 5.1. Given a deformation of $\varphi$ as in (4.1), the full torsion $\tilde{T}$ of the new $G_{2}$-structure $\tilde{\varphi}$ is given by

$$
\begin{aligned}
\tilde{T}_{a}^{\tilde{m}}= & \left(\frac{\operatorname{det} g}{\operatorname{det} \tilde{g}}\right)^{\frac{1}{2}}\left(T_{a}{ }^{m}+\frac{1}{24} T_{a}{ }^{e} \psi_{e b c d} * \chi^{m b c d}+\frac{1}{24} \psi^{m b c d} \nabla_{a} \chi_{b c d}\right. \\
& \left.+\frac{1}{24} \nabla_{a} \chi_{b c d} * \chi^{m b c d}\right)-\frac{1}{2} \delta \Gamma_{a b}^{e} \tilde{\varphi}_{e}^{\tilde{m} \tilde{b}}
\end{aligned}
$$


with $\delta \Gamma_{a b}^{e}$ given by (4.23).

Proof. Starting from (3.5) for $\tilde{\varphi}$ and $\tilde{\psi}$, we get

$$
\begin{aligned}
\tilde{T}_{a}^{\tilde{m}} & =\frac{1}{24}\left(\tilde{\nabla}_{a} \tilde{\varphi}_{b c d}\right) \tilde{\psi}^{\tilde{m} \tilde{b} \tilde{c} \tilde{d}} \\
& =\frac{1}{24}\left(\nabla_{a} \tilde{\varphi}_{b c d}-3 \delta \Gamma_{a b}^{e} \tilde{\varphi}_{c d e}\right) \tilde{\psi}^{\tilde{m} \tilde{b} \tilde{c} \tilde{d}} \\
& =\frac{1}{24}\left(\left(\frac{\operatorname{det} g}{\operatorname{det} \tilde{g}}\right)^{\frac{1}{2}} \nabla_{a} \tilde{\varphi}_{b c d} * \tilde{\varphi}^{m b c d}-3 \delta \Gamma_{a b}^{e} \tilde{\varphi}_{c d e} \tilde{\psi}^{\tilde{m} \tilde{b} \tilde{c} \tilde{d}}\right) .
\end{aligned}
$$

We can write

$$
\tilde{\varphi}_{c d e} \tilde{\psi}^{\tilde{m} \tilde{b} \tilde{d} \tilde{d}}=4 \tilde{\varphi}_{e}^{\tilde{m} \tilde{b}}
$$

and we can expand

$$
\begin{aligned}
\nabla_{a} \tilde{\varphi}_{b c d} * \tilde{\varphi}^{m b c d}= & \left(\nabla_{a} \varphi_{b c d}+\nabla_{a} \chi_{b c d}\right)\left(\psi^{m b c d}+* \chi^{m b c d}\right) \\
= & 24 T_{a}{ }^{m}+T_{a}{ }^{e} \psi_{e b c d} * \chi^{m b c d} \\
& +\psi^{m b c d} \nabla_{a} \chi_{b c d}+\nabla_{a} \chi_{b c d} * \chi^{m b c d} .
\end{aligned}
$$

Hence the result.

The torsion classes $W_{i}$ were originally defined by the $G_{2}$-structure $\varphi$, so once we have deformed $\varphi$ to $\tilde{\varphi}$ we will also get new torsion classes. Denote the new space by $\tilde{W}$ which splits as

$$
\tilde{W}=\tilde{W}_{1} \oplus \tilde{W}_{7} \oplus \tilde{W}_{14} \oplus \tilde{W}_{27} .
$$

The new torsion $\tilde{T}$ should now split as

$$
\left.\tilde{T}_{a b}=\tilde{\tau}_{1} \tilde{g}_{a b}+\left(\tilde{\tau}_{7}^{\tilde{\#}}\right\lrcorner \tilde{\varphi}\right)_{a b}+\left(\tilde{\tau}_{14}\right)_{a b}+\left(\tilde{\tau}_{27}\right)_{a b} .
$$

Note that $\tilde{\tau}_{7}^{\#}$ refers to the vector obtained from the 1 -form $\tilde{\tau}_{7}$ by raising indices using the deformed inverse metric $\tilde{g}^{-1}$. In general, determining these new torsion components $\tilde{\tau}_{i}$ is quite complicated. First we would have to lower one of the indices in (5.31) using $\tilde{g}$ and extract the different components. It is however easy to extract the the $\tilde{W}_{1}$-component directly from (5.31) by just contracting the indices.

LEMMA 5.2. The $\tilde{W}_{1}$-component $\tilde{\tau}_{1}$ of the deformed torsion $\tilde{T}$ is given by $\tilde{\tau}_{1}=\left(\frac{\operatorname{det} g}{\operatorname{det} \tilde{g}}\right)^{\frac{1}{2}}\left(\tau_{1}+\frac{1}{168} T_{a}^{e} \psi_{e b c d} * \chi^{a b c d}+\frac{1}{168} \psi^{a b c d} \nabla_{a} \chi_{b c d}+\frac{1}{168} \nabla_{a} \chi_{b c d} * \chi^{a b c d}\right)$.

Proof. Contracting the indices in (5.31) we get

$$
\begin{aligned}
\tilde{T}_{a}^{\tilde{a}}= & \left(\frac{\operatorname{det} g}{\operatorname{det} \tilde{g}}\right)^{\frac{1}{2}}\left(T_{a}{ }^{a}+\frac{1}{24} T_{a}{ }^{e} \psi_{e b c d} * \chi^{a b c d}+\frac{1}{24} \psi^{a b c d} \nabla_{a} \chi_{b c d}\right. \\
& \left.+\frac{1}{24} \nabla_{a} \chi_{b c d} * \chi^{a b c d}\right)-\frac{1}{2} \delta \Gamma_{a b}^{e} \tilde{\varphi}_{e}^{\tilde{a} \tilde{b}} .
\end{aligned}
$$


Note that since the Christoffel symbols are symmetric in the bottom two indices, $\delta \Gamma_{a}^{e} b$ is also symmetric in $a$ and $b$, so

$$
\delta \Gamma_{a b b}^{e} \tilde{\varphi}_{e}^{\tilde{a} \tilde{b}}=0 .
$$

Since $\tilde{T}_{a}^{\tilde{a}}=7 \tilde{\tau}_{1}$, we get the result.

Consider now what happens to $\tilde{T}_{a n}$ with lowered indices.

$$
\begin{aligned}
\tilde{T}_{a n}= & \tilde{T}_{a}^{\tilde{m}} \tilde{g}_{m n}=\left(\frac{\operatorname{det} g}{\operatorname{det} \tilde{g}}\right)\left(T_{a}^{m}+\frac{1}{24} T_{a}{ }^{e} \psi_{e b c d} * \chi^{m b c d}+\frac{1}{24} \psi^{m b c d} \nabla_{a} \chi_{b c d}\right. \\
& \left.+\frac{1}{24} \nabla_{a} \chi_{b c d} * \chi^{m b c d}\right) s_{m n}-\frac{1}{2} \delta \Gamma_{a b}^{e} \tilde{\varphi}_{e n}^{\tilde{b}} .
\end{aligned}
$$

Now using the expression for $\delta \Gamma_{a b}^{e}(4.23)$, we get

$$
\begin{aligned}
\delta \Gamma_{a b}^{e} \tilde{\varphi}_{e n}^{\tilde{b}}= & \frac{1}{2}\left(\frac{\operatorname{det} g}{\operatorname{det} \tilde{g}}\right)^{\frac{1}{2}}\left(\tilde{g}^{\tilde{e} \tilde{d}} \tilde{\varphi}_{e n}^{\tilde{b}}\left(\nabla_{b} s_{a d}+\nabla_{a} s_{b d}-\nabla_{d} s_{a b}\right)\right. \\
& \left.-\frac{1}{9}\left(\delta_{a}^{e} \delta_{b}^{f}+\delta_{b}^{e} \delta_{a}^{f}-\tilde{g}_{a b} \tilde{g}^{\tilde{e} \tilde{f}}\right) \tilde{\varphi}_{e n}^{\tilde{b}} \tilde{g}^{\tilde{p} \tilde{q}} \nabla_{f} s_{p q}\right) .
\end{aligned}
$$

Simplifying further, we eventually get

$$
\begin{aligned}
\delta \Gamma_{a b b}{ }_{b} \tilde{\varphi}_{e n}^{\tilde{b}}= & \left(\frac{\operatorname{det} g}{\operatorname{det} \tilde{g}}\right)^{\frac{1}{2}}\left(\tilde{\varphi}_{n}^{\tilde{b} \tilde{d}} \nabla_{b} s_{a d}-\frac{1}{9} \tilde{\varphi}_{a n} \tilde{f} \tilde{g}^{\tilde{p} \tilde{q}} \nabla_{f} s_{p q}\right) \\
= & \left(\frac{\operatorname{det} g}{\operatorname{det} \tilde{g}}\right)^{\frac{1}{2}} \tilde{\varphi}_{c}^{\tilde{b} \tilde{d}}\left(\delta_{n}^{c} \nabla_{b} s_{a d}-\frac{1}{9} \delta_{a}^{c} \tilde{g}_{b n} \tilde{g}^{\tilde{p} \tilde{q}} \nabla_{d} s_{p q}\right) \\
= & \frac{1}{4}\left(\frac{\operatorname{det} g}{\operatorname{det} \tilde{g}}\right)\left(4 \varphi_{c}^{b d}+\varphi_{c m n} * \chi^{m n b d}+\chi_{c m n} \psi^{m n b d}+\chi_{c m n} * \chi^{m n b d}\right) \times \\
& \times\left(\delta_{n}^{c} \nabla_{b} s_{a d}-\frac{1}{9} \delta_{a}^{c} \tilde{g}_{b n} \tilde{g}^{\tilde{p} \tilde{q}} \nabla_{d} s_{p q}\right) .
\end{aligned}
$$

Thus, overall we have

$$
\begin{aligned}
\tilde{T}_{a n}= & \frac{1}{24}\left(\frac{\operatorname{det} g}{\operatorname{det} \tilde{g}}\right)\left(24 T_{a}^{m}+T_{a}^{e} \psi_{e b c d} * \chi^{m b c d}+\psi^{m b c d} \nabla_{a} \chi_{b c d}+\nabla_{a} \chi_{b c d} * \chi^{m b c d}\right) \times \\
& \times s_{m n}-\frac{1}{8}\left(\frac{\operatorname{det} g}{\operatorname{det} \tilde{g}}\right)\left(4 \varphi_{c}^{b d}+\varphi_{c p q} * \chi^{p q b d}+\chi_{c p q} \psi^{p q b d}+\chi_{c p q} * \chi^{p q b d}\right) \times \\
& \times\left(\delta_{n}^{c} \nabla_{b} s_{a d}-\frac{1}{9} \delta_{a}^{c} \tilde{g}_{b n} \tilde{g}^{\tilde{p} \tilde{q}} \nabla_{d} s_{p q}\right) .
\end{aligned}
$$

In the particular case of conformal deformations we can simply plug in $\chi, s$ and $\tilde{g}$ as in (4.10), (4.11) and (4.13) into (5.34) and obtain the deformed torsion.

Proposition 5.3. Let $(\varphi, g)$ be a $G_{2}$-structure with torsion $T$. Then define $(\tilde{\varphi}, \tilde{g})$ to be a new $G_{2}$-structure given by a conformal transformation of $(\varphi, g)$ :

$$
\begin{aligned}
\tilde{\varphi} & =f^{3} \varphi \\
\tilde{g} & =f^{2} g .
\end{aligned}
$$


Then the full torsion tensor $\tilde{T}$ is given by

$$
\tilde{T}=f T-d f\lrcorner \varphi .
$$

Thus from Proposition 5.3 we see that a conformal transformation simply scales the torsion classes in $W_{1}, W_{14}$ and $W_{27}$, while the change to $W_{7}$ is more complicated. Therefore, the only conformally invariant torsion classes are the ones that contain a $W_{7}$ component. This was previously shown in [6] and [13] but here we have an explicit expression for the torsion from which this conclusion follows trivially.

The expression (5.36) also shows that if the $W_{7}$ component of the original torsion is an exact form, then it is possible to remove this component by applying a particular conformal transformation. Note that this implies that the class $W_{1} \oplus W_{7}$ is conformal to the class $W_{1}$. As we know from the torsion conditions, if $\tau_{1}$ is never 0 , then

$$
\tau_{7}=d\left(\log \left|\tau_{1}\right|\right)
$$

Hence in order to remove this torsion component, need

$$
d\left(\log \left|\tau_{1}\right|\right)=\frac{1}{f} d f
$$

hence,

$$
f=\frac{\left|\tau_{1}\right|}{C}
$$

is a solution for a constant $C$. The original torsion is

$$
\left.T=\tau_{1} g+\frac{1}{f} d f\right\lrcorner \varphi
$$

so, under the change (5.36), the new torsion will become

$$
\tilde{T}=\frac{\tau_{1}^{2}}{C} g
$$

However, under the transformation

$$
\varphi \longrightarrow\left(\frac{\left|\tau_{1}\right|}{C}\right)^{3} \varphi
$$

the metric changes as

$$
g \longrightarrow\left(\frac{\tau_{1}}{C}\right)^{2} g
$$

Hence in terms of the new metric, the new torsion is

$$
\tilde{T}=C \tilde{g}
$$

and so the constant $C$ is in fact equal to the new $W_{1}$ torsion component $\tilde{\tau}_{1}$. Thus the conformal transformation (5.37) reduces the class $W_{1} \oplus W_{7}$ to $W_{1}$. Conversely, a conformal transformation of the $W_{1}$ class will result in $W_{1} \oplus W_{7}$. Since $G_{2}$-structures in the $W_{1}$ class are sometimes called nearly $G_{2}$ or nearly parallel, the $G_{2}$-structures in the strict $W_{1} \oplus W_{7}$ class are referred to as conformally nearly parallel. If $W_{1}=0$, 
then we just have the $W_{7}$ class. In this case, we just know that $\tau_{7}$ is closed. So by Poincaré Lemma, we can at least locally find a function $h$ such that $d h=\tau_{7}$. By taking a conformal transformation with $f=e^{h}$, we can thus locally fully remove the torsion. Hence the $W_{7}$ class is sometimes called locally conformally parallel.

For the class $W_{1} \oplus W_{7}$ we can also explicitly write out the Ricci curvature.

Corollary 5.4. Suppose the 3 -form $\varphi$ defines a $G_{2}$-structure with torsion contained in the class $W_{1} \oplus W_{7}$. The the Ricci curvature of the corresponding metric is given by

$$
R_{a b}=\left(\nabla^{c}\left(\tau_{7}\right)_{c}+5\left(\tau_{7}\right)^{c}\left(\tau_{7}\right)_{c}+6 \tau_{1}^{2}\right) g_{a b}-5\left(\tau_{7}\right)_{a}\left(\tau_{7}\right)_{b}+5 \nabla_{a}\left(\tau_{7}\right)_{b} .
$$

Proof. In the general expression for the Ricci curvature, (3.18), substitute

$$
T_{a b}=\tau_{1} g_{a b}+\left(\tau_{7}\right)^{c} \varphi_{c a b} .
$$

We then get

$$
\begin{aligned}
R_{a b}= & \left(\nabla^{c}\left(\tau_{7}\right)_{c}+5\left(\tau_{7}\right)^{c}\left(\tau_{7}\right)_{c}+6 \tau_{1}^{2}\right) g_{a b}-5\left(\tau_{7}\right)_{a}\left(\tau_{7}\right)_{b}+5 \nabla_{b}\left(\tau_{7}\right)_{a} \\
& -\psi_{a b}{ }^{c d} \nabla_{c}\left(\tau_{7}\right)_{d}+\varphi_{a b}{ }^{c} \nabla_{c} \tau_{1}-\tau_{1} \varphi_{a b}{ }^{c}\left(\tau_{7}\right)_{c} .
\end{aligned}
$$

However using the fact that $d \tau_{7}=0$, and hence that $\nabla_{a}\left(\tau_{7}\right)_{b}$ is symmetric, and moreover that $\nabla_{c} \tau_{1}=\tau_{1}\left(\tau_{7}\right)_{c}$, we obtain (5.38).

6. Torsion for $\Lambda_{7}$ deformations. Now consider in detail the case when we have a deformation in $\Lambda_{7}$. Here we have

$$
h_{a b}=v^{c} \varphi_{c a b} .
$$

Then,

$$
\begin{aligned}
\chi_{b c d} & =v^{e} \psi_{b c d e} \\
* \chi_{m n p q} & =4 v_{[m} \varphi_{n p q]} .
\end{aligned}
$$

So we take a $G_{2}$-structure $\varphi$ and deform it to

$$
\tilde{\varphi}=\varphi+v^{e} \psi_{b c d e} .
$$

Recall that for convenience we have

$$
M=|v|^{2}
$$

then, as we know from (4.19),

$$
s_{a b}=g_{a b}(1+M)-v_{a} v_{b}
$$

and

$$
\begin{aligned}
\left(\frac{\operatorname{det} \tilde{g}}{\operatorname{det} g}\right)^{\frac{1}{2}} & =(1+M)^{\frac{2}{3}} \\
\tilde{g}_{a b} & =(1+M)^{-\frac{2}{3}}\left(g_{a b}(1+M)-v_{a} v_{b}\right) \\
\tilde{g}^{\tilde{a} \tilde{b}} & =(1+M)^{-\frac{1}{3}}\left(g^{a b}+v^{a} v^{b}\right) \\
\tilde{\varphi}_{a}^{\tilde{b} \tilde{c}} & =(1+M)^{-\frac{2}{3}}\left(\varphi_{a}^{b c}-\chi_{a}^{b c}+v^{b} v_{m} \varphi_{a}^{c m}-v^{c} v_{m} \varphi_{a}^{b m}\right) .
\end{aligned}
$$


The last equation comes from (4.20). Note that the deformed metric defined above is always positive definite. To see this, suppose $\xi^{a}$ is some vector, then

$$
\tilde{g}_{a b} \xi^{a} \xi^{b}=\left(1+|v|^{2}\right)^{-\frac{2}{3}}\left(|\xi|^{2}+|v|^{2}|\xi|^{2}-\left(v_{a} \xi^{a}\right)^{2}\right) \geq 0
$$

since $\left(v_{a} \xi^{a}\right)^{2} \leq|v|^{2}|\xi|^{2}$. Therefore, under such a deformation, the 3-form $\tilde{\varphi}$ is always a positive 3 -form, and thus indeed defines a $G_{2}$-structure.

Now let us use the expression for deformed torsion (5.35) to write it down in terms of $v$. First, we have

$$
\nabla_{d} s_{p q}=2 g_{p q} v_{m}\left(\nabla_{d} v^{m}\right)-\left(\nabla_{d} v_{p}\right) v_{q}-\left(\nabla_{d} v_{q}\right) v_{p}
$$

and thus,

$$
\begin{aligned}
\delta_{n}^{c} \nabla_{b} s_{a d}-\frac{1}{9} \delta_{a}^{c} \tilde{g}_{b n} \tilde{g}^{\tilde{p} \tilde{q}} \nabla_{d} s_{p q}= & \nabla_{e} s_{p q}\left(\delta_{n}^{c} \delta_{b}^{e} \delta_{a}^{p} \delta_{d}^{q}-\frac{1}{9} \delta_{a}^{c} \delta_{d}^{e} \tilde{g}_{b n} \tilde{g}^{\tilde{p} \tilde{q}}\right) \\
= & (1+M)^{-1} \nabla_{e} s_{p q}\left((1+M) \delta_{n}^{c} \delta_{b}^{e} \delta_{a}^{p} \delta_{d}^{q}\right. \\
& \left.-\frac{1}{9} \delta_{a}^{c} \delta_{d}^{e}\left(g_{b n}(1+M)-v_{b} v_{n}\right)\left(g^{p q}+v^{p} v^{q}\right)\right) .
\end{aligned}
$$

So, overall, we have

$$
\begin{aligned}
\tilde{T}_{a n}= & \frac{1}{24}(1+M)^{-\frac{4}{3}}\left(\left(24 T_{a}{ }^{m}+T_{a}{ }^{e} \psi_{e b c d} * \chi^{m b c d}+\psi^{m b c d} \nabla_{a} \chi_{b c d}\right.\right. \\
& \left.+\nabla_{a} \chi_{b c d} * \chi^{m b c d}\right) s_{m n}-3(1+M)^{-1}\left(\varphi_{c}{ }^{b d}-\chi_{c}{ }^{b d}+v^{b} v_{m} \varphi_{c}{ }^{d m}-v^{d} v_{m} \varphi_{c}{ }^{b m}\right) \times \\
& \left.\times \nabla_{e} s_{p q}\left((1+M) \delta_{n}^{c} \delta_{b}^{e} \delta_{a}^{p} \delta_{d}^{q}-\frac{1}{9} \delta_{a}^{c} \delta_{d}^{e}\left(g_{b n}(1+M)-v_{b} v_{n}\right)\left(g^{p q}+v^{p} v^{q}\right)\right)\right) .
\end{aligned}
$$

It makes sense to expand $\nabla v$ also in terms of $G_{2}$-representations:

$$
\nabla_{a} v_{b}=v_{1} g_{a b}+\left(v_{7}\right)^{c} \varphi_{c a b}+\left(v_{14}\right)_{a b}+\left(v_{27}\right)_{a b}
$$

where $v_{14} \in \Lambda_{14}^{2}$ and $v_{27}$ is traceless symmetric. Together with the similar expansion of $T_{a n}$ (3.6), after some manipulations, we obtain:

THEOREM 6.1. Given a $G_{2}$-structure $\varphi$ with full torsion tensor $T_{a b}$, a deformation of $\varphi$ which lies $\Lambda_{7}^{3}$ given by $\varphi \longrightarrow \varphi+v_{e} \psi_{\text {bcd }}$ e results in a new $G_{2}$-structure $\tilde{\varphi}$ with torsion tensor $\tilde{T}_{\text {an }}$ given by

$$
\begin{aligned}
\tilde{T}_{a n}= & (1+M)^{-\frac{4}{3}}\left(v_{1}\left(v_{a} v_{n}-(1+M) g_{a n}\right)-\frac{4}{3}(1+M) v_{1} \varphi_{a n m} v^{m}\right. \\
& -\left(1+\frac{4}{3} M\right) \varphi_{a n m}\left(v_{7}\right)^{m}-\frac{1}{3} \psi_{a n m p} v^{m}\left(v_{7}\right)^{p}+\frac{5}{3} v_{a} \varphi_{n m p} v^{m}\left(v_{7}\right)^{p}+\frac{4}{3} v_{n} \varphi_{a m p} v^{m}\left(v_{7}\right)^{p} \\
& +\frac{1}{3}\left(v_{7}\right)^{m} v_{m} \varphi^{p}{ }_{a n} v_{p}+\frac{1}{3} v_{n}\left(v_{7}\right)_{a}+\frac{8}{3} v_{a}\left(v_{7}\right)_{n}-(1+M)\left(v_{14}\right)_{a n} \\
& -2 v_{m}\left(v_{14}\right)_{[a}^{m} v_{n]}+\frac{1}{3} \varphi_{a n m}\left(v_{14}\right)^{m p} v_{p}+\frac{1}{3} \psi_{a n m p} v_{q} v^{m}\left(v_{14}\right)^{p q}-(1+M)\left(v_{27}\right)_{a n} \\
& +v_{m}\left(v_{27}\right)^{m}{ }_{a} v_{n}-(1+M) \varphi^{m p}{ }_{a}\left(v_{27}\right)_{p n} v_{m}-\frac{1}{3} \varphi_{a n m}\left(v_{27}\right)^{m p} v_{p} \\
& \left.+\frac{1}{3} \psi_{a n m p} v^{m}\left(v_{27}\right)^{p q} v_{q}+v_{a} \varphi_{n m p} v^{m}\left(v_{27}\right)^{p q} v_{q}-\frac{1}{3} \varphi_{a n}{ }^{m} v_{m}\left(v_{27}\right)^{p q} v_{p} v_{q}\right) \\
& +(1+M)^{-\frac{1}{3}}\left(\tau_{1} g_{a n}+\tau_{1} \varphi_{a n}^{m} v_{m}+\varphi_{a n m}\left(\tau_{7}\right)^{m}+v_{a}\left(\tau_{7}\right)_{n}-g_{a n}\left(\tau_{7}\right)^{m} v_{m}\right. \\
& \left.+\psi_{a n m p}\left(\tau_{7}\right)^{m} v^{p}+\left(\tau_{14}\right)_{a n}-\varphi_{n m p} v^{m}\left(\tau_{14}\right)^{p}{ }_{a}+\left(\tau_{27}\right)_{a n}+\varphi_{n m p} v^{m}\left(\tau_{27}\right)_{a}\right)
\end{aligned}
$$


From this we can also extract the individual components of $\tilde{T}_{a n}$ in the representations of $G_{2}$. So first we have the component of $\tilde{T}_{a n}$ in $\tilde{W}_{1}$ :

$$
\begin{aligned}
\tilde{\tau}_{1} & =\frac{1}{7} \tilde{T}_{a b} \tilde{g}^{\tilde{a} \tilde{b}}=(1+M)^{-\frac{1}{3}}\left(\tilde{T}_{a b} g^{a b}+v^{a} v^{b} \tilde{T}_{a b}\right) \\
& =(1+M)^{-\frac{2}{3}}\left(\left(1+\frac{1}{7} M\right) \tau_{1}-v_{1}-\frac{6}{7}\left(\tau_{7}\right)^{a} v_{a}+\frac{3}{7}\left(v_{7}\right)^{a} v_{a}+\frac{1}{7}\left(\tau_{27}\right)_{a b} v^{a} v^{b}\right) .
\end{aligned}
$$

The 7-dimensional component is given by

$$
\left(\tilde{\tau}_{7}\right)_{c}=\frac{1}{6} \tilde{T}_{a b} \tilde{\varphi}_{c}^{\tilde{a} \tilde{b}}=\frac{1}{6}(1+M)^{-\frac{2}{3}} \tilde{T}_{a b}\left(\varphi^{a b}{ }_{c}-v^{m} \psi_{c m}^{a b}+v^{a} v_{m} \varphi_{c}^{b m}-v^{b} v_{m} \varphi^{a m}{ }_{c}\right)
$$

where we have used (4.20). Now using the expression for $\tilde{T}_{a b}$ (6.44), after some manipulations, we obtain

$$
\begin{aligned}
\left(\tilde{\tau}_{7}\right)_{c}= & \left(\tau_{7}\right)_{c}-\frac{1}{6} \varphi_{c}^{a b}\left(\tau_{7}\right)_{a} v_{b}-\frac{1}{6} v^{a}\left(\tau_{27}\right)_{a c}-\frac{1}{6} v^{a}\left(\tau_{14}\right)_{a c} \\
& +\frac{v_{c}}{6(1+M)}\left(\left(\tau_{27}\right)_{a b} v^{a} v^{b}+6 \tau_{1}-6\left(\tau_{7}\right)_{a} v^{a}-8 v_{1}+3\left(v_{7}\right)_{a} v^{a}\right) \\
& -\frac{1}{6(1+M)}\left(3(M+2)\left(v_{7}\right)_{c}+v^{a}\left(v_{27}\right)_{a c}+\varphi_{c a}^{b} v^{a}\left(v_{27}\right)_{b d} v^{d}+3 \varphi_{c a b} v^{a}\left(v_{7}\right)^{b}\right) .
\end{aligned}
$$

Let us now find the $\tilde{W}_{14}$ component. We have $\tilde{T}_{[a n]} \in \Lambda^{2}$, so

$$
\pi_{14}\left(\tilde{T}_{[a n]}\right)=\frac{2}{3} \tilde{T}_{[a n]}-\frac{1}{6} \tilde{T}_{m p} \tilde{\psi}^{\tilde{m} \tilde{p}}{ }_{a n} .
$$

The skew-symmetric part of (6.44) is given by:

$$
\begin{aligned}
\tilde{T}_{[a n]}= & (1+M)^{-\frac{4}{3}}\left(-\frac{4}{3}(1+M) v_{1} \varphi_{a n m} v^{m}-\left(1+\frac{4}{3} M\right) \varphi_{a n m}\left(v_{7}\right)^{m}\right. \\
& -\frac{1}{3} \psi_{a n m p} v^{m}\left(v_{7}\right)^{p}+v_{[a} \varphi_{n] m p} v^{m}\left(v_{7}\right)^{p}+\frac{1}{3}\left(v_{7}\right)^{m} v_{m} \varphi_{a n}^{p} v_{p}+\frac{7}{3} v_{[a}\left(v_{7}\right)_{n]} \\
& -(1+M)\left(v_{14}\right)_{a n}-2 v_{m}\left(v_{14}\right)_{[a}^{m} v_{n]}+\frac{1}{3} \varphi_{a n m}\left(v_{14}\right)^{m p} v_{p}+\frac{1}{3} \psi_{a n m p} v_{q} v^{m}\left(v_{14}\right)^{p q} \\
& +v_{m}\left(v_{27}\right)_{[a}^{m} v_{n]}-(1+M) \varphi_{[a}^{m p}\left(v_{27}\right)_{n] p} v_{m}-\frac{1}{3} \varphi_{a n m}\left(v_{27}\right)^{m p} v_{p} \\
& \left.+\frac{1}{3} \psi_{a n m p} v^{m}\left(v_{27}\right)^{p q} v_{q}+v_{[a} \varphi_{n] m p} v^{m}\left(v_{27}\right)^{p q} v_{q}-\frac{1}{3} \varphi_{a n}{ }^{m} v_{m}\left(v_{27}\right)^{p q} v_{p} v_{q}\right) \\
& +(1+M)^{-\frac{1}{3}}\left(\tau_{1} \varphi^{m}{ }_{a n} v_{m}+\varphi_{a n m}\left(\tau_{7}\right)^{m}+v_{[a}\left(\tau_{7}\right)_{n]}+\psi_{a n m p}\left(\tau_{7}\right)^{m} v^{p}\right. \\
& \left.+\left(\tau_{14}\right)_{a n}+\varphi_{m p[a} v^{m}\left(\tau_{14}\right)_{n]}^{p}-\varphi_{m p[a} v^{m}\left(\tau_{27}\right)_{n]}^{p}\right) .
\end{aligned}
$$

Now note that

$$
\begin{aligned}
\tilde{\psi}_{a n}^{\tilde{m} \tilde{p}}=\tilde{\psi}^{\tilde{m} \tilde{q} \tilde{r} \tilde{g}} \tilde{g}_{q a} \tilde{g}_{n r}= & (1+M)^{-2}\left(\psi^{m p q r}+4 v^{[m} \varphi^{p q r]}\right) \times \\
& \times\left(g_{a q}(1+M)-v_{a} v_{q}\right)\left(g_{n r}(1+M)-v_{n} v_{r}\right) .
\end{aligned}
$$


Hence the 14-dimensional component is

$$
\begin{aligned}
& \left(\tilde{\tau}_{14}\right)_{a n} \\
= & (1+M)^{-\frac{4}{3}}\left(\frac{10}{3}\left(v_{7}\right)_{[a} v_{n]}+\frac{4}{3} v_{[a} \varphi^{m p}{ }_{n]} v_{m}\left(v_{7}\right)_{p}-\left(\frac{5}{6}+\frac{1}{2} M\right) \psi_{a n}^{m p} v_{m}\left(v_{7}\right)_{p}\right. \\
& +\frac{1}{3}\left(v_{7}\right)_{m} v^{m} v_{p} \varphi_{a n}^{p}-\frac{1}{3} M\left(v_{7}\right)_{m} \varphi_{a n}^{m}-(1+M)\left(v_{14}\right)_{a n}-2 v_{m}\left(v_{14}\right)^{m}{ }_{[a} v_{n]} \\
& +\frac{1}{3} \varphi_{a n}^{m} v_{p}\left(v_{14}\right)_{m}^{p}+\frac{1}{3} \psi_{a n}^{m p} v_{m}\left(v_{14}\right)_{p q} v^{q}-\frac{1}{3} \varphi_{a n}^{m} v_{m}\left(v_{27}\right)_{p q} v^{p} v^{q} \\
& +(M+1) \varphi_{[a}^{m p}\left(v_{27}\right)_{n] p} v_{m}+\frac{1}{6}(M-1) \varphi_{a n}^{m}\left(v_{27}\right)_{m}^{p} v_{p} \\
& \left.+\frac{2}{3} v_{m}\left(v_{27}\right)_{[a}^{m} v_{n]}-\frac{4}{3} \varphi_{[a}^{m p} v_{n]} v_{m}\left(v_{27}\right)_{p q} v^{q}+\frac{1}{3} \psi_{a n}^{m p} v_{m}\left(v_{27}\right)_{p q} v^{q}\right) \\
& +(1+M)^{-\frac{1}{3}}\left(-\frac{1}{6} M \varphi_{a n}^{m}\left(\tau_{7}\right)_{m}+\frac{1}{6} \psi_{a n}^{m p}\left(\tau_{7}\right)_{m} v_{p}-\frac{1}{3} \varphi_{[a}^{m p} v_{n]}\left(\tau_{7}\right)_{m} v_{p}+\frac{2}{3} v_{[a}\left(\tau_{7}\right)_{n]}\right. \\
& +\frac{1}{6} \varphi_{a n}^{m} v_{m} v_{p}\left(\tau_{7}\right)^{p}+\left(\tau_{14}\right)_{a n}+\frac{1}{6} \psi_{a n}^{m p}\left(\tau_{14}\right)_{m}^{q} v_{p} v_{q}-\frac{1}{3} \varphi_{a n}^{m} v_{p}\left(\tau_{14}\right)_{m}^{p} \\
& \left.-\varphi^{m p}{ }_{[a}\left(\tau_{27}\right)_{n] p} v_{m}+\frac{1}{6} \varphi_{a n}^{m}\left(\tau_{27}\right)_{m}^{p} v_{p}+\frac{1}{6} \psi_{a n}^{m p}\left(\tau_{27}\right)_{m}^{q} v_{p} v_{q}\right) .
\end{aligned}
$$

Finally, the component in $\tilde{W}_{27}$ is now given by

$$
\left(\tilde{\tau}_{27}\right)_{a n}=\tilde{T}_{(a n)}-\tau_{1} \tilde{g}_{a n}
$$

where $\tilde{T}_{(a n)}$ is the symmetric part of (6.44):

$$
\begin{aligned}
\tilde{T}_{(a n)}= & (1+M)^{-\frac{4}{3}}\left(v_{1}\left(v_{a} v_{n}-(1+M) g_{a n}\right)+3 v_{(a} \varphi_{n) m p} v^{m} v_{7}^{p}\right. \\
& +3 v_{(a}\left(v_{7}\right)_{n)}-(1+M)\left(v_{27}\right)_{a n}+v_{m}\left(v_{27}\right)_{(a}^{m} v_{n)} \\
& -(1+M) \varphi^{m p}\left({ }_{a}\left(v_{27}\right)_{n) p} v_{m}+v_{(a} \varphi_{n) m p} v^{m}\left(v_{27}\right)^{p q} v_{q}\right) \\
& +(1+M)^{-\frac{1}{3}}\left(\tau_{1} g_{a n}+v_{(a}\left(\tau_{7}\right)_{n)}-g_{a n}\left(\tau_{7}\right)^{m} v_{m}\right. \\
& \left.-\varphi_{m p(a} v^{m}\left(\tau_{14}\right)_{n)}^{p}+\left(\tau_{27}\right)_{a n}+\varphi_{m p(a} v^{m}\left(\tau_{27}\right)_{n)}^{p}\right)
\end{aligned}
$$

and

$$
\begin{aligned}
\tilde{\tau}_{1} \tilde{g}_{a n}= & (1+M)^{-\frac{4}{3}}\left((1+M) g_{a n}-v_{a} v_{n}\right) \times \\
& \times\left(\left(1+\frac{1}{7} M\right) \tau_{1}-v_{1}-\frac{6}{7}\left(\tau_{7}\right)^{m} v_{m}+\frac{3}{7}\left(v_{7}\right)^{m} v_{m}-\frac{1}{7}\left(\tau_{27}\right)^{m p} v_{m} v_{p}\right) .
\end{aligned}
$$

Thus overall, we have

$$
\begin{aligned}
& \left(\tilde{\tau}_{27}\right)_{a n} \\
= & (1+M)^{-\frac{4}{3}}\left(-\frac{3}{7}\left((1+M) g_{a n}-v_{a} v_{n}\right)\left(v_{7}\right)^{m} v_{m}+3 v_{(a} \varphi_{n) m p} v^{m}\left(v_{7}\right)^{p}\right. \\
& +3 v_{(a}\left(v_{7}\right)_{n)}-(1+M)\left(v_{27}\right)_{a n}+v_{m}\left(v_{27}\right)_{(a}^{m} v_{n)}-(1+M) \varphi_{(a}^{m p}\left(v_{27}\right)_{n) p} v_{m} \\
& \left.+v_{(a} \varphi_{n) m p} v^{m}\left(v_{27}\right)^{p q} v_{q}+\left(1+\frac{1}{7} M\right) \tau_{1} v_{a} v_{n}-\frac{6}{7}\left(\tau_{7}\right)^{m} v_{m} v_{a} v_{n}+\frac{1}{7}\left(\tau_{27}\right)^{m p} v_{m} v_{p} v_{a} v_{n}\right) \\
& +(1+M)^{-\frac{1}{3}}\left(-\frac{1}{7} M \tau_{1} g_{a n}+v_{(a}\left(\tau_{7}\right)_{n)}-\frac{1}{7} g_{a n}\left(\tau_{7}\right)^{m} v_{m}\right. \\
& \left.-\varphi_{m p(a} v^{m}\left(\tau_{14}\right)_{n)}^{p}+\left(\tau_{27}\right)_{a n}+\varphi_{m p(a} v^{m}\left(\tau_{27}\right)_{n)}^{p}-\frac{1}{7}\left(\tau_{27}\right)^{m p} v_{m} v_{p} g_{a n}\right) .
\end{aligned}
$$


The expressions (6.45), (6.47), (6.50) and (6.52) give us the components of the new torsion $\tilde{T}$ in $\tilde{W}_{1}, \tilde{W}_{7}, \tilde{W}_{14}$ and $\tilde{W}_{27}$, respectively. As we can see these expressions are quite complicated, so for a generic deformation vector $v$, in general we would obtain

THEOREM 6.2. Given a $G_{2}$-structure $\varphi$ with full torsion tensor $T_{a b}$, a deformation of $\varphi$ which lies $\Lambda_{7}^{3}$ given by $\varphi \longrightarrow \varphi+v_{e} \psi_{b c d}$ e results in a new $G_{2}$-structure $\tilde{\varphi}$ with a torsion tensor $\tilde{T}_{a n}$ if and only if $v$ satisfies the differential equation

$$
\left.\nabla v=v_{1} g+\left(v_{7}\right)\right\lrcorner \varphi+v_{14}+v_{27}
$$

where the components $v_{1}, v_{7}, v_{14}$ and $v_{27}$ of $\nabla v$ are given by

$$
\begin{gathered}
v_{1}=\tau_{1}-\frac{3}{7}\left(\tau_{7}\right)_{a} v^{a}-\frac{1}{7} \frac{(7+3 M)}{(1+M)^{\frac{1}{3}}} \tilde{\tau}_{1}-\frac{3}{7}\left(\tilde{\tau}_{7}\right)_{a} v^{a} \\
+\frac{1}{14}(1+M)^{\frac{1}{3}}\left(\tilde{\tau}_{27}\right)_{a b} v^{a} v^{b} \\
\left(v_{7}\right)^{c}=\left(\tau_{7}\right)^{c}-\frac{1}{3} \tau_{1} v^{c}+\frac{1}{3} \varphi^{c}{ }_{a b}\left(\tau_{7}\right)^{a} v^{b}-\frac{1}{6}\left(\tau_{14}\right)_{a}{ }^{c} v^{a}-\frac{1}{3}\left(\tau_{27}\right)_{a}{ }_{a}^{c} v^{a} \\
+\frac{4}{3} \frac{\tilde{\tau}_{1}}{(1+M)^{\frac{1}{3}}} v^{c}-\left(\tilde{\tau}_{7}\right)^{c}-\frac{1}{2} \varphi^{c}{ }_{a b}\left(\tilde{\tau}_{7}\right)^{a} v^{b}+\frac{1}{6}(1+M)^{\frac{1}{3}}\left(\tilde{\tau}_{27}\right)_{a}{ }^{c} v^{a}
\end{gathered}
$$

$\left(v_{14}\right)_{a b}$

$=\frac{1}{(M+9)}\left(\frac{4}{3}(M-27)\left(\tau_{7}\right)_{[a} v_{b]}-\frac{1}{3}(M-27) \psi_{a b}^{m n}\left(\tau_{7}\right)_{m} v_{n}-4 M \varphi_{a b}^{m}\left(\tau_{7}\right)_{m}\right.$

$+4\left(\tau_{7}\right)^{m} v_{m} \varphi_{a b}^{n} v_{n}-24 \varphi_{[a}^{m n} v_{b]}\left(\tau_{7}\right)_{m} v_{n}+\frac{1}{2}(M+2)(M+9)\left(\tau_{14}\right)_{a b}$

$+\frac{1}{2}(M+9) \varphi_{a}^{m n} \varphi_{b}^{p q} v_{m} v_{p}\left(\tau_{14}\right)_{n q}+(M-7) v_{m}\left(\tau_{14}\right)_{[a}^{m} v_{b]}$

$+8 \varphi_{[a}^{m n} v_{b]} v_{n} v_{p}\left(\tau_{14}\right)^{p}{ }_{m}-\frac{4}{3} M v_{m}\left(\tau_{14}\right)_{n}^{m} \varphi_{a b}^{n}+4 \psi_{a b}^{m n} v_{p}\left(\tilde{\tau}_{14}\right)_{m}^{p} v_{n}$

$+16 v_{m}\left(\tau_{27}\right)_{[a}^{m} v_{b]}-\frac{4}{3} v_{m} \varphi_{a b}^{m}\left(\tau_{27}\right)_{n p} v^{n} v^{p}+\frac{4}{3} M v_{m}\left(\tau_{27}\right)^{m}{ }_{n} \varphi_{a b}^{n}-4 \psi_{a b}^{m n} v_{p}\left(\tau_{27}\right)_{m}^{p} v_{n}$

$+8 \varphi_{[a}^{m n} v_{b]} v_{n} v_{p}\left(\tau_{27}\right)_{m}^{p}+\left(\frac{1}{6}(M+17)(1+M)^{\frac{1}{3}}\left(\tilde{\tau}_{27}\right)_{m n} v^{m} v^{n}-4\left(\tilde{\tau}_{7}\right)_{m} v^{m}\right) v_{p} \varphi_{a b}^{p}$

$+24 \varphi_{[a}^{m n} v_{b]}\left(\tilde{\tau}_{7}\right)_{m} v_{n}-2(M-15)\left(\tilde{\tau}_{7}\right)_{[a} v_{b]}+\frac{1}{2}(M-15) \psi_{a b}^{m n}\left(\tilde{\tau}_{7}\right)_{m} v_{n}$

$+4 M \varphi_{a b}^{m}\left(\tilde{\tau}_{7}\right)_{m}-(1+M)^{\frac{1}{3}}(M+9)\left(\left(\tilde{\tau}_{14}\right)_{a b}+v^{m} \varphi_{m n[a}\left(\tilde{\tau}_{27}\right)_{b]}^{n}\right)$

$+16(1+M)^{-\frac{2}{3}} v_{m}\left(\tilde{\tau}_{14}\right)_{[a}^{m} v_{b]}+8(1+M)^{-\frac{2}{3}} \varphi_{[a}^{m n} v_{b]} v_{n} v_{p}\left(\tilde{\tau}_{14}\right)_{m}^{p}$

$+(M-3)(1+M)^{-\frac{2}{3}} v_{m}\left(\tilde{\tau}_{14}\right)^{m}{ }_{n} \varphi_{a b}^{n}-4(1+M)^{-\frac{2}{3}} \psi_{a b}^{m n} v_{p}\left(\tilde{\tau}_{14}\right)_{m}^{p} v_{n}$

$-8(1+M)^{\frac{1}{3}} \varphi_{[a}^{m n} v_{b]} v_{n} v_{p}\left(\tilde{\tau}_{27}\right)_{m}^{p}+8(1+M)^{\frac{1}{3}} v_{m}\left(\tilde{\tau}_{27}\right)_{[a}^{m} v_{b]}$

$\left.+\frac{1}{6}(7 M-9)(1+M)^{\frac{1}{3}} v_{m}\left(\tilde{\tau}_{27}\right)_{n}^{m} \varphi_{a b}^{n}-4(1+M)^{\frac{1}{3}} \psi_{a b}^{m n} v_{p}\left(\tilde{\tau}_{27}\right)_{m}^{p} v_{n}\right)$ 


$$
\begin{aligned}
& \left(v_{27}\right)_{a b} \\
= & \left(\tau_{27}\right)_{a b}+4\left(\tau_{7}\right)_{(a} v_{b)}+\left(4(1+M)^{-\frac{1}{3}} \tilde{\tau}_{1}-\frac{1}{2}(1+M)^{-\frac{2}{3}}\left(\tilde{\tau}_{27}\right)_{m n} v^{m} v^{n}\right) v_{a} v_{b} \\
& -\frac{1}{7}\left(4\left(\tau_{7}\right)_{m} v^{m}-3\left(\tilde{\tau}_{7}\right)_{m} v^{m}-4 M(1+M)^{-\frac{1}{3}} \tilde{\tau}_{1}-\frac{1}{2}(1+M)^{-\frac{1}{3}}\left(\tilde{\tau}_{27}\right)_{m n} v^{m} v^{n}\right) g_{a b} \\
& -3\left(\tilde{\tau}_{7}\right)_{(a} v_{b)}-\varphi_{(a}^{m n}\left(\tilde{\tau}_{14}\right)_{b) n} v_{m}-\frac{1}{2}(1+M)^{-\frac{2}{3}} \varphi_{a}^{m n} \varphi_{b}^{p q}\left(\tilde{\tau}_{27}\right)_{m p} v_{n} v_{q} \\
& -(1+M)^{-\frac{2}{3}}\left(\frac{1}{2}(2+M)\left(\tilde{\tau}_{27}\right)_{a b}+v_{m}\left(\tilde{\tau}_{27}\right)_{(a}^{m} v_{b)}+v^{m} \varphi_{m n(a}\left(\tilde{\tau}_{27}\right)_{b)}^{n}\right. \\
& \left.+\varphi_{(a}^{m n} v_{b)} v_{n} v_{p}\left(\tilde{\tau}_{27}\right)_{m}^{p}\right) .
\end{aligned}
$$

Proof. In order to obtain the equations which the components $v_{1}, v_{7}, v_{14}$ and $v_{27}$ of $\nabla v$ must satisfy, first note that the expressions for $\tilde{\tau}_{1}, \tilde{\tau}_{7}, \tilde{\tau}_{14}$ and $\tilde{\tau}_{27}$ that are given by (6.45), (6.46), (6.50) and (6.52), are all linear in $\nabla v$. We then solve for $v_{1}$, $\left(v_{7}\right)^{c},\left(v_{14}\right)_{a b}$ and $\left(v_{27}\right)_{a b}$ in terms of the original torsion components $\tau_{1}, \tau_{7}, \tau_{14}$ and $\tau_{27}$ and the new torsion components $\tilde{\tau}_{1}, \tilde{\tau}_{7}, \tilde{\tau}_{14}$ and $\tilde{\tau}_{27}$. Pointwise we have the same number of variables as equations, so generically there is a solution.

For convenience, let us denote the left hand sides of equations (6.45), (6.46), (6.50) and (6.52), by $\hat{\tau}_{1}, \hat{\tau}_{7}, \hat{\tau}_{14}$ and $\hat{\tau}_{27}$, respectively. Hence these equations can be rewritten as

$$
\begin{aligned}
\tilde{\tau}_{1} & =\hat{\tau}_{1} \\
\tilde{\tau}_{7} & =\hat{\tau}_{7} \\
\tilde{\tau}_{14} & =\hat{\tau}_{14} \\
\tilde{\tau}_{27} & =\hat{\tau}_{27} .
\end{aligned}
$$

Let us first look at the $\tilde{\tau}_{1}$ equation. Note that the expression for $\tilde{\tau}_{1}$ contains the scalars $v_{1}$ and $\left(v_{7}\right)^{a} v_{a}$. So in order to find $v_{1}$, we would also need to find $\left(v_{7}\right)^{a} v_{a}$. We can get another equation that has $\left(v_{7}\right)^{a} v_{a}$ by constructing the scalar $\left(\tilde{\tau}_{7}\right)^{c} v_{c}$. However that now also has the scalar $\left(v_{27}\right)^{a b} v_{a} v_{b}$. So we would need another equation - this time from by $\left(\tilde{\tau}_{27}\right)_{a b} v^{a} v^{b}$. Now we can solve the system

$$
\begin{aligned}
\tilde{\tau}_{1}= & \hat{\tau}_{1}=(1+M)^{-\frac{2}{3}}\left(-v_{1}+\frac{3}{7}\left(v_{7}\right)^{a} v_{a}+\left(1+\frac{1}{7} M\right) \tau_{1}\right. \\
& \left.-\frac{6}{7}\left(\tau_{7}\right)^{a} v_{a}+\frac{1}{7}\left(\tau_{27}\right)^{a b} v_{a} v_{b}\right) \\
\left(\tilde{\tau}_{7}\right)^{c} v_{c}= & \left(\hat{\tau}_{7}\right)^{c} v_{c}=(1+M)^{-1}\left(-\frac{4}{3} M v_{1}-\left(v_{7}\right)^{a} v_{a}-\frac{1}{6}\left(v_{27}\right)^{a b} v_{a} v_{b}\right. \\
& \left.+M \tau_{1}+\left(\tau_{7}\right)^{a} v_{a}-\frac{1}{6}\left(\tau_{27}\right)^{a b} v_{a} v_{b}\right) \\
\left(\tilde{\tau}_{27}\right)_{a b} v^{a} v^{b}= & \left(\hat{\tau}_{27}\right)_{a b} v^{a} v^{b}=(1+M)^{-\frac{4}{3}}\left(\frac{18}{7} M\left(v_{7}\right)^{a} v_{a}-\left(v_{27}\right)^{a b} v_{a} v_{b}\right. \\
& \left.+\frac{6}{7} M^{2} \tau_{1}+\frac{6}{7} M\left(\tau_{7}\right)^{a} v_{a}+\left(1+\frac{6}{7} M\right)\left(\tau_{27}\right)^{a b} v_{a} v_{b}\right) .
\end{aligned}
$$

We have three equations, and the three variables, $v_{1},\left(v_{7}\right)^{a} v_{a}$ and $\left(v_{27}\right)_{a b} v^{a} v^{b}$, which are independent. The determinant of this system is proportional to $(M+1)$, but 
$M=|v|^{2}>0$, so we always have a solution. Solving, we get the solution (6.53b) for $v_{1}$ and also solutions for $\left(v_{7}\right)^{a} v_{a}$ and $\left(v_{27}\right)_{a b} v^{a} v^{b}$.

Note that we could have also considered $\left(\tilde{\tau}_{27}\right)_{a b} g^{a b}$. However, since $\tilde{\tau}_{27}$ is traceless with respect to $\tilde{g}^{a b}=(1+M)^{-\frac{2}{3}}\left(g^{a b}+v^{a} v^{b}\right)$,

$$
\left(\tilde{\tau}_{27}\right)_{a b} g^{a b}=-\left(\tilde{\tau}_{27}\right)_{a b} v^{a} v^{b}
$$

so we would get no new independent equation.

Next, we look at the $\tilde{\tau}_{7}$ equation. We now have expressions for $\left(v_{7}\right)^{a} v_{a}$ and $\left(v_{27}\right)^{a b} v_{a} v_{b}$, so we can replace any instances of these scalars by the solutions of the above scalar equations. Our remaining variables are now $\left(v_{7}\right)^{c},\left(v_{14}\right)_{a}{ }^{c} v^{a},\left(v_{27}\right)_{a}{ }^{c} v^{a}$, $\varphi^{c}{ }_{a b}\left(v_{7}\right)^{a} v^{b}, \varphi^{c}{ }_{a b}\left(v_{14}\right)^{e b} v^{a} v_{e}$ and $\varphi_{a b}^{c}\left(v_{27}\right)^{b e} v^{a} v_{e}$. To solve for these variables, we construct six equations

$$
\begin{aligned}
& \left(\tilde{\tau}_{7}\right)_{a}=\left(\hat{\tau}_{7}\right)_{a} \\
& \left(\tilde{\tau}_{14}\right)_{a b} v^{a}=\left(\hat{\tau}_{14}\right)_{a b} v^{a} \\
& \left(\tilde{\tau}_{27}\right)_{a b} v^{a}=\left(\hat{\tau}_{27}\right)_{a b} v^{a} \\
& \varphi_{b c}^{a}\left(\tilde{\tau}_{7}\right)^{b} v^{c}=\varphi^{a}{ }_{b c}\left(\hat{\tau}_{7}\right)^{b} v^{c} \\
& \varphi^{a}{ }_{b c}\left(\tilde{\tau}_{14}\right)^{b}{ }_{d} v^{d} v^{c}=\varphi^{a}{ }_{b c}\left(\hat{\tau}_{14}\right)_{d}^{b} v^{d} v^{c} \\
& \varphi^{a}{ }_{b c}\left(\tilde{\tau}_{27}\right)^{b}{ }_{d} v^{d} v^{c}=\varphi_{b c}^{a}\left(\hat{\tau}_{27}\right)_{d}^{b} v^{d} v^{c} .
\end{aligned}
$$

The left hand side of each of these equations is now some function of $v, \tau_{1}, \tau_{7}, \tau_{14}$ and $\tau_{27}$ constructed from the expressions for $\tilde{\tau}_{1}, \tilde{\tau}_{7}, \tilde{\tau}_{14}$ and $\tilde{\tau}_{27}$ and with any instances of $v_{1},\left(v_{7}\right)^{a} v_{a}$ and $\left(v_{27}\right)^{a b} v_{a} v_{b}$ replaced by the solutions of equations (6.54). It turns out that we do not get any new variables, and so we get six equations for six variables. The determinant of this system is positive, so we can solve this, and in particular, get the solution for $\left(v_{7}\right)^{c}(6.53 \mathrm{~b})$. We also get solutions for the other vectors constructed above.

Now we can look at the last two equations - $\left(\tilde{\tau}_{14}\right)_{a b}=\left(\hat{\tau}_{14}\right)_{a b}$ and $\left(\tilde{\tau}_{27}\right)_{a b}=$ $\left(\hat{\tau}_{27}\right)_{a b}$. We now have solutions for scalars and vectors, so we can substitute them into these equations. Then, the variables in the first equation are skew-symmetric quantities, and in the second equation we have symmetric quantities.

In the $\tilde{\tau}_{14}$ equation the quantities are $\left(v_{14}\right)_{a b}$ and $\varphi_{[a}^{c d}\left(v_{27}\right)_{b] d} v_{c}$, while in the $\tilde{\tau}_{27}$ equation we have $\left(v_{27}\right)_{a b}$ and $\varphi_{(a}^{c d}\left(v_{27}\right)_{b) d} v_{c}$. Hence we can construct quantities $\varphi_{[a}^{c d}\left(\tilde{\tau}_{27}\right)_{b] d} v_{c}$ and $\varphi_{(a}^{c d}\left(\tilde{\tau}_{27}\right)_{b) d} v_{c}$ which give us one extra equation for both skewsymmetric and symmetric quantities. For the skew-symmetric equations we get no new variables, thus our equations are

$$
\begin{aligned}
& \left(\tilde{\tau}_{14}\right)_{a b}=\left(\hat{\tau}_{14}\right)_{a b} \\
& \varphi_{[a}^{c d}\left(\tilde{\tau}_{27}\right)_{b] d} v_{c}=\varphi_{[a}^{c d}\left(\hat{\tau}_{27}\right)_{b] d} v_{c} .
\end{aligned}
$$

Here we solve for $\left(v_{14}\right)_{a b}$ and $\varphi^{c d}{ }_{[a}\left(v_{27}\right)_{b] d} v_{c}$, and immediately get the solution (6.53d). It can be checked that this expression does indeed give a 2-form lying in $\Lambda_{14}^{2}$.

Going back to the symmetric equations, from $\varphi^{c d}{ }_{(a}\left(\tilde{\tau}_{27}\right)_{b) d} v_{c}$ we get a new symmetric variable $\varphi_{a}{ }^{c d} \varphi_{b}{ }^{e f} v_{c} v_{e}\left(v_{27}\right)_{d f}$. We then construct the quantity $\varphi_{a}{ }^{c d} \varphi_{b}{ }^{e f} v_{c} v_{e}\left(\tilde{\tau}_{27}\right)_{d f}$ and get no new variables. Therefore, the symmetric equations 
are

$$
\begin{aligned}
& \left(\tilde{\tau}_{27}\right)_{a b}=\left(\hat{\tau}_{27}\right)_{a b} \\
& \varphi^{c d}\left(\tilde{\tau}_{27}\right)_{b) d} v_{c}=\varphi^{c d}{ }_{(a}\left(\hat{\tau}_{27}\right)_{b) d} v_{c} \\
& \varphi_{a}{ }^{c d} \varphi_{b}{ }^{e f} v_{c} v_{e}\left(\tilde{\tau}_{27}\right)_{d f}=\varphi_{a}{ }^{c d} \varphi_{b}{ }^{e f} v_{c} v_{e}\left(\hat{\tau}_{27}\right)_{d f}
\end{aligned}
$$

where we solve for $\left(v_{27}\right)_{a b}, \varphi^{c d}{ }_{(a}\left(v_{27}\right)_{b) d} v_{c}$ and $\varphi_{a}{ }^{c d} \varphi_{b}{ }^{e f} v_{c} v_{e}\left(v_{27}\right)_{d f}$. We have three equations with three variables, and the determinant is again positive, so we solve it and get the solution (6.53e) for $\left(v_{27}\right)_{a b}$. Note that it is always traceless, hence indeed always corresponds to the component in the 27 -dimensional representation.

Basically, Theorem 6.2 gives us that $v$ satisfies the differential equation

$$
\nabla v=F(T, \tilde{T}, v)
$$

where $F$ is a 2-tensor-valued function that is linear in $T$ and $\tilde{T}$, and non-linear in $v$. Its components are given by $(6.53 \mathrm{~b})-(6.53 \mathrm{e})$. Note that if $\tilde{T}=0$, then $F$ is in fact a rational function of $v$. This can be observed directly from (6.53). This is an overdetermined PDE, and the standard approach is to differentiate it further and apply the Ricci identity, with the hope of obtaining some constraints. Differentiating (6.58) we get

$$
\begin{aligned}
\nabla_{a} \nabla_{b} v_{c} & =\nabla_{a} F_{b c} \\
& =\frac{\partial F_{b c}}{\partial T_{m n}} \nabla_{a} T_{m n}+\frac{\partial F_{b c}}{\partial \tilde{T}_{m n}} \nabla_{a} \tilde{T}_{m n}+\frac{\partial F_{b c}}{\partial v_{m}} \nabla_{a} v_{m} \\
& =\frac{\partial F_{b c}}{\partial T_{m n}} \nabla_{a} T_{m n}+\frac{\partial F_{b c}}{\partial \tilde{T}_{m n}} \nabla_{a} \tilde{T}_{m n}+\frac{\partial F_{b c}}{\partial v_{m}} F_{a m}
\end{aligned}
$$

Antisymmetrizing the covariant derivatives, we get

$$
\begin{aligned}
-R_{c a b}^{d} v_{d}= & \frac{\partial F_{b c}}{\partial T_{m n}} \nabla_{a} T_{m n}-\frac{\partial F_{a c}}{\partial T_{m n}} \nabla_{b} T_{m n}+\frac{\partial F_{b c}}{\partial \tilde{T}_{m n}} \nabla_{a} \tilde{T}_{m n} \\
& -\frac{\partial F_{a c}}{\partial \tilde{T}_{m n}} \nabla_{b} \tilde{T}_{m n}+\frac{\partial F_{b c}}{\partial v_{m}} F_{a m}-\frac{\partial F_{a c}}{\partial \tilde{T}_{m n}} \nabla_{b} \tilde{T}_{m n}
\end{aligned}
$$

This expression gives an algebraic constraint on $v$ and involves only the curvature, the old and the new torsion and the derivatives of the torsion. Note that by projecting to the 7 component of the Riemann curvature, we can get an expression that only involves the vector $v$, the old and new torsion and their derivatives. Moreover, we can also apply the conditions on the derivatives of torsion components from Proposition 3.3 in order to relate some of the torsion derivatives to the torsion components themselves. Alternatively, to eliminate the curvature term, we can antisymmetrize over indices $a$, $b$ and $c$, and apply the Bianchi identity. This is however equivalent to noting that $d^{2} v^{b}=0$ is always satisfied. However from the decomposition of $\nabla v$, we have

$$
\left.d v^{b}=2\left(v_{7}\right)\right\lrcorner \varphi+2 v_{14} .
$$

Therefore, we must have

$$
\left.d\left(\left(v_{7}\right)\right\lrcorner \varphi+v_{14}\right)=0 .
$$


Using the expressions (6.53b) and (6.53d) for $v_{7}$ and $v_{14}$, respectively, and then applying (6.58) to eliminate the derivatives of $v$, we get a relationship between $v$, the torsion components and the derivatives of the torsion components. Again, some of the torsion derivatives can also be eliminated using Proposition 3.3. In the general case, the resulting expressions are extremely long, and not very helpful, so we will consider individual torsion classes in order to gain more insight.

As we have noted above, the expressions (6.53) for the components of $\nabla v$ become much more manageable when we set $\tilde{T}=0$. So we will first consider deformations to a torsion-free $G_{2}$-structure. The simplest case is when the original torsion also vanishes.

Corollary 6.3. Suppose the 3 -form $\varphi$ defines a torsion-free $G_{2}$-structure, then a deformation of $\varphi$ which lies in $\Lambda_{7}^{3}$ and is given by $\varphi \longrightarrow \varphi+v^{e} \psi_{\text {bcde }}$ results in a new torsion-free $G_{2}$-structure $\tilde{\varphi}$ if and only if

$$
\nabla v=0 .
$$

Proof. We get this immediately by setting all the torsion components to zero in (6.53b) to (6.53e) in Theorem 6.2.

NOTATION 6.4 .

LEMma 6.5. If $(\varphi, g)$ is a torsion-free $G_{2}$-structure, then for a 3 -form $\left.\chi=v\right\lrcorner \psi$ the following are equivalent
1. $\nabla v=0$
2. $\nabla \chi=0$
3. $d \chi=0$ and $d^{*} \chi=0$.

Proof. Starting from the first statement, if $v$ is parallel, then since $\nabla \psi=0$ in the torsion-free case, we have $\nabla \chi=0$ and hence $d \chi=0$ and $d^{*} \chi=0$. Now suppose conversely, $d \chi=0$ and $d^{*} \chi=0$. Let us first work out $d^{*} \chi$ We then get:

$$
\left(d^{*} \chi\right)_{b c}=\nabla_{m} v_{n} \psi_{b c}^{m n} .
$$

From [14] (and adjusting for our sign convention) we know however that for a 2 -form $\omega$

$$
\psi_{m n b c} \omega^{b c}=4\left(\pi_{7} \omega\right)_{m n}-2\left(\pi_{14} \omega\right)_{m n} .
$$

Hence if $\psi_{m n b c} \omega^{b c}=0$, then $\pi_{7} \omega$ and $\pi_{14} \omega$ must vanish individually, and thus $\omega=0$. Applying this to (6.62) we get that $d v^{b}=0$, that is the skew-symmetric part of $\nabla v$. Now consider the type decomposition of the exterior derivative $d \chi$ is

$$
\begin{aligned}
\pi_{1} d \chi & =\frac{4}{7}\left(\nabla_{m} v^{m}\right) \psi \\
\pi_{7} d \chi & =-\frac{1}{2}\left(\nabla_{m} v_{n} \varphi^{m n p}\right) \wedge \varphi \\
\pi_{1 \oplus 27} d \chi & =-3 * \mathrm{i}_{\varphi}\left(\nabla_{(a} v_{b)}+\frac{1}{3}\left(\nabla_{m} v^{m}\right) g_{a b}\right) .
\end{aligned}
$$

If $d \chi=0$, then each component must vanish individually. Hence we also get that the symmetric part of $\nabla v$ vanishes. Therefore, overall we have that $\nabla v=0$. 
Thus, using Lemma 6.5, the result in Corollary 6.3 is equivalent to saying that $d \chi=0$ and $d * \chi=0$ for $\chi=v^{e} \psi_{b c d e}$. This is however exactly the same condition as the one for an infinitesimal deformation.

TheOREM 6.6. Suppose $(\varphi, g)$ is a $G_{2}$-structure on a closed, compact manifold $M$. Consider a deformation of the $G_{2}$-structure $\varphi$ given by

$$
\varphi \longrightarrow \varphi+v^{e} \psi_{b c d e}
$$

If the torsion $T$ lies in the class $W_{1} \oplus W_{7}$, then this deformation results in a torsionfree $G_{2}$-structure if and only if $T=0$ and $\nabla v=0$.

Proof. If $T=0$, from Corollary 6.3, we know that the deformation (6.66) results in a torsion-free $G_{2}$-structure if and only if $\nabla v=0$. So assume now $T \neq 0$.

Now let us assume that $T \in W_{1} \subset W_{1} \oplus W_{7}$ and suppose the deformation (6.66) results in $\tilde{T}=0$. Thus here we have $\tau_{7}=\tau_{14}=\tau_{27}=0$ and $\tilde{\tau}_{1}=\tilde{\tau}_{7}=\tilde{\tau}_{14}=\tilde{\tau}_{27}=0$. Also, Then from Theorem 6.2, we have

$$
\nabla_{a} v_{b}=\tau_{1} g_{a b}-\frac{1}{3} \tau_{1} v^{c} \varphi_{c a b}
$$

and in particular,

$$
\left.d v^{b}=-\frac{2}{3} \tau_{1} v\right\lrcorner \varphi
$$

Now, using the fact that in this case, $\tau_{1}$ is constant, the consistency condition $d^{2} v^{b}=0$ is equivalent to either $\tau_{1}=0$ or

$$
d(v\lrcorner \varphi)=0
$$

Consider $\left.\pi_{1}(d(v\lrcorner \varphi)\right)$. Then expanding and using the fact that $\nabla \varphi=\tau_{1} \psi$, it is easy to see that

$$
\begin{aligned}
\left.\pi_{1}(d(v\lrcorner \varphi)\right) & =\frac{3}{7}\left(\nabla^{m} v_{m}\right) \varphi_{a b c} \\
& =3 \tau_{1} \varphi_{a b c}
\end{aligned}
$$

where have applied (6.67) to get the second line. So we must have $\tau_{1}=0$, which gives a contradiction. Hence there are no deformations from torsion class $W_{1}$ to $W_{0}$.

Next we assume that $T \in W_{7} \subset W_{1} \oplus W_{7}$, so that only $\tau_{7}$ is non-vanishing. In this case,

$$
\begin{aligned}
\nabla_{a} v_{b}= & (M+9)^{-1}\left(-g_{a b}\left(\tau_{7}\right)_{c} v^{c}+3(1+M) v_{b}\left(\tau_{7}\right)_{a}+(33+M) v_{a}\left(\tau_{7}\right)_{b}\right. \\
& -3 \varphi_{a b}^{c}\left(\tau_{7}\right)_{c}(M-3)+4\left(\tau_{7}\right)_{c} v^{c} \varphi_{a b d} v^{d}-24 \varphi_{d[a}^{c} v_{b]}\left(\tau_{7}\right)_{c} v^{d} \\
& \left.+12 \psi_{d a b}^{c}\left(\tau_{7}\right)_{c} v^{d}\right)
\end{aligned}
$$

and correspondingly we can also get $d v^{b}$ from this. As before, we consider $d\left(d v^{b}\right)$ and the projections of it on to $\Lambda_{1}^{3}, \Lambda_{7}^{3}$ and $\Lambda_{27}^{3}$. Let $\xi_{1}$ be the scalar corresponding to the $\Lambda_{1}^{3}$ projection, $\xi_{7}$ - the vector corresponding to the $\Lambda_{7}^{3}$ projection and $\xi_{27}$ - the symmetric 2 -tensor corresponding to the $\Lambda_{27}^{3}$ component. As before, we can obtain 
scalars $\left(\xi_{7}\right)_{a} v^{a}$ and $\left(\xi_{27}\right)_{a b} v^{a} v^{b}$. Hence we get three scalar equations

$$
\begin{aligned}
0= & 16(M-15)\left(\left(\tau_{7}\right)_{a} v^{a}\right)^{2}-6\left(3 M^{2}-34 M+27\right)\left(\tau_{7}\right)^{a}\left(\tau_{7}\right)_{a} \\
& -(M+9)^{2} \nabla^{a}\left(\tau_{7}\right)_{a} \\
0= & \frac{4(M-3)\left(\left(\tau_{7}\right)_{a} v^{a}\right)^{2}}{M+9}-\frac{9 M(M+3)\left(\tau_{7}\right)^{a}\left(\tau_{7}\right)_{a}}{M+9}+\left(\nabla_{a}\left(\tau_{7}\right)_{b}\right) v^{a} v^{b} \\
& -M \nabla^{a}\left(\tau_{7}\right)_{a} \\
0= & \frac{6(1+M)(M-39)\left(\left(\tau_{7}\right)_{a} v^{a}\right)^{2}}{M+9}-\frac{4 M(5 M-3)(M-3)\left(\tau_{7}\right)^{a}\left(\tau_{7}\right)_{a}}{M+9} \\
& +2(M-3)\left(\nabla_{a}\left(\tau_{7}\right)_{b}\right) v^{a} v^{b}-3 M(1+M) \nabla^{a}\left(\tau_{7}\right)_{a} .
\end{aligned}
$$

We can solve these equations to get $\nabla^{a}\left(\tau_{7}\right)_{a},\left(\nabla_{a}\left(\tau_{7}\right)_{b}\right) v^{a} v^{b}$ and $\left(\tau_{7}\right)^{a}\left(\tau_{7}\right)_{a}=\left|\tau_{7}\right|^{2}$ in terms of $\left(\left(\tau_{7}\right)_{a} v^{a}\right)^{2}=\left\langle\tau_{7}, v\right\rangle^{2}$. So in particular, we get

$$
\left|\tau_{7}\right|^{2}=\frac{3\left\langle\tau_{7}, v\right\rangle^{2}\left(3 M^{2}-10 M+51\right)}{\left(7 M^{2}-66 M-9\right) M} .
$$

Further, from $\xi_{7}^{d}=0, \varphi_{a b c} v^{b} \xi_{7}^{c}=0,\left(\xi_{27}\right)_{m n} v^{n}=0$ and $\varphi_{a b c}\left(\xi_{27}\right)^{b}{ }_{n} v^{n} v^{c}=0$, we actually find that

$$
v=\frac{M}{\left\langle\tau_{7}, v\right\rangle} \tau_{7}
$$

and after contracting with $\tau_{7}$, we get

$$
\left|\tau_{7}\right|^{2}=\frac{\left\langle\tau_{7}, v\right\rangle^{2}}{M}
$$

Comparing (6.72) and (6.74), we get

$$
\left\langle\tau_{7}, v\right\rangle^{2}(M+9)^{2}=0 .
$$

Hence $\left\langle\tau_{7}, v\right\rangle=0$ and so must have $\tau_{7}=0$. Therefore, there are no deformations from $W_{7}$ to $W_{0}$.

Finally, suppose $T_{a b}$ lies in the strict class $W_{1} \oplus W_{7}$, so that the $W_{1}$ component of the torsion is $\tau_{1}$ and the $W_{7}$ component is $\tau_{7}$. In this case, from Theorem 6.2 , we have

$$
\begin{aligned}
\nabla_{a} v_{b}= & \left(\tau_{1}-\left(\tau_{7}\right)_{c} v^{c}\right) g_{a b}+\frac{1}{(M+9)}\left(-3(M-3)\left(\tau_{7}\right)_{c} \varphi_{a b}^{c}\right. \\
& -(M+33) v_{a}\left(\tau_{7}\right)_{b}+3(1+M)\left(\tau_{7}\right)_{a} v_{b} \\
& -\frac{1}{3} v^{c} \varphi_{c a b}\left(9 \tau_{1}+\tau_{1} M-12\left(\tau_{7}\right)_{d} v^{d}\right) \\
& \left.+12 v_{a} \varphi_{b}^{c d}\left(\tau_{7}\right)_{c} v_{d}-12 v_{b} \varphi_{a}^{c d}\left(\tau_{7}\right)_{c} v_{d}+12\left(\tau_{7}\right)_{c} v_{d} \psi_{a b}^{c d}\right) .
\end{aligned}
$$

Following the general procedure outlined above, we used Maple to expand the necessary condition (6.61). Again, as before, we consider the projections of $d\left(d v^{b}\right)$. As outlined above we first consider the $\pi_{1}, \pi_{7}$ and $\pi_{27}$ projections of $\left.d\left(\left(v_{7}\right)\right\lrcorner \varphi+v_{14}\right)$.

Denote by $\xi_{1}$ the scalar corresponding to the $\Lambda_{1}^{3}$ component, let $\xi_{7}$ and $\xi_{27}$ be the vector and symmetric tensor components. Then by considering the equations $\xi_{1}=0$, 
$\left(\xi_{7}\right)^{a} v_{a}=0$ and $\left(\xi_{27}\right)_{m n} v^{m} v^{n}=0$, we can express $\left(\nabla_{a}\left(\tau_{7}\right)_{b}\right) v^{a} v^{b}, \nabla^{a}\left(\tau_{7}\right)_{a}$ and $\left|\tau_{7}\right|^{2}$ in terms of $M, \tau_{1}$ and $\left\langle\tau_{7}, v\right\rangle$. In particular, we find that

$$
\begin{aligned}
\left|\tau_{7}\right|^{2}= & \frac{3\left\langle\tau_{7}, v\right\rangle^{2}\left(3 M^{2}-10 M+51\right)}{\left(7 M^{2}-66 M-9\right) M}-\frac{4}{3} \frac{\tau_{1}\left\langle\tau_{7}, v\right\rangle(M+9)^{2}}{\left(7 M^{2}-66 M-9\right)} \\
& +\frac{2}{9} \frac{\tau_{1}^{2} M(M+9)^{2}}{7 M^{2}-66 M-9} .
\end{aligned}
$$

Further, we can consider the vector equations $\xi_{7}^{d}=0, \varphi_{a b c} v^{b} \xi_{7}^{c}=0,\left(\xi_{27}\right)_{m n} v^{n}=0$ and $\varphi_{a b c}\left(\xi_{27}\right)^{b}{ }_{n} v^{n} v^{c}=0$. From these, in particular, we find

$$
\tau_{7}=\frac{\left\langle\tau_{7}, v\right\rangle}{M} v
$$

So as before, we get

$$
\left|\tau_{7}\right|^{2}=\frac{\left\langle\tau_{7}, v\right\rangle^{2}}{M} .
$$

Now if we equate (6.76) and (6.78), and then solve for $\left\langle\tau_{7}, v\right\rangle$, we obtain an expression for $\left\langle\tau_{7}, v\right\rangle$ in terms of $\tau_{1}, \tau_{7}$ and $v$.

$$
\left\langle\tau_{7}, v\right\rangle=\frac{M \tau_{1}}{3}
$$

Hence,

$$
v=\frac{3}{\tau_{1}} \tau_{7}
$$

and,

$$
\begin{aligned}
M & =\frac{9}{\tau_{1}^{2}}\left|\tau_{7}\right|^{2} \\
\left\langle\tau_{7}, v\right\rangle & =\frac{3}{\tau_{1}}\left|\tau_{7}\right|^{2} .
\end{aligned}
$$

Next, from equations $\left(\xi_{27}\right)_{a b}=0, \varphi_{(a}^{c d}\left(\xi_{27}\right)_{b) d} v_{c}=0$ and $\varphi_{a}^{c d} \varphi_{b}^{e f} v_{c} v_{e}\left(\xi_{27}\right)_{d f}=$ 0 , we finally obtain an expression for $\nabla_{a}\left(\tau_{7}\right)_{b}$. Using (6.80) and (6.81) to completely eliminate $v$ from the resulting expression, we overall get:

$$
\nabla \tau_{7}=\left(\frac{1}{3} \tau_{1}^{2}-\left|\tau_{7}\right|^{2}\right) g+5 \tau_{7} \otimes \tau_{7} .
$$

By first considering the trace of this, we find that we get the condition

$$
\nabla_{a} \tau_{7}^{a}+2\left(\tau_{7}\right)_{a}\left(\tau_{7}\right)^{a}-\frac{7}{3} \tau_{1}^{2}=0 .
$$

Recall however, that a $G_{2}$-structure in the strict torsion class $W_{1} \oplus W_{7}$ has

$$
\tau_{7}=\nabla\left(\log \tau_{1}\right) .
$$

So we can rewrite $(6.83)$ as

$$
\Delta\left(\log \tau_{1}\right)-2\left|\nabla\left(\log \tau_{1}\right)\right|^{2}+\frac{7}{3} \tau_{1}^{2}=0
$$


Now note that if we let $F=\tau_{1}^{2}$, then

$$
\Delta F=-\frac{14}{3} F^{2} .
$$

However, if we integrate both sides over the whole manifold $M$, and apply Stokes's Theorem we find that the integral of the left hand side is zero since $M$ is closed, while the integral of right hand side is the negative $L_{2}$-norm of $F$. Hence we must have $F=0$ and this implies that both $\tau_{1}$ and $\tau_{7}$ vanish. Therefore we cannot have a deformation from $W_{1} \oplus W_{7}$ into $W_{0}$.

THEOREM 6.7. There is no deformation of the form (6.66) within the strict torsion class $W_{1}$.

Proof. We consider a $G_{2}$-structure $(\varphi, g)$ where the only non-zero component of torsion $T$ is $\tau_{1}$. Suppose (6.66) gives a deformation to a $G_{2}$-structure $(\tilde{\varphi}, \tilde{g})$ with torsion $\tilde{T}$ with the only non-zero component being $\tilde{\tau}_{1}$. Then from Theorem 6.2 ,

$$
\begin{aligned}
\nabla_{a} v_{b}= & \left(\tau_{1}-(1+M)^{\frac{2}{3}} \tilde{\tau}_{1}\right) g_{a b}+4(1+M)^{-\frac{1}{3}} \tilde{\tau}_{1} v_{a} v_{b} \\
& -\frac{1}{3} v^{c} \varphi_{c a b}\left(\tau_{1}-4(1+M)^{-\frac{1}{3}} \tilde{\tau}_{1}\right)
\end{aligned}
$$

and in particular,

$$
\left.d v^{b}=-\frac{2}{3} v\right\lrcorner \varphi\left(\tau_{1}-4(1+M)^{-\frac{1}{3}} \tilde{\tau}_{1}\right) .
$$

Then we take $d\left(d v^{b}\right)$, and decompose it into $\Lambda_{1}^{3}, \Lambda_{7}^{3}$ and $\Lambda_{27}^{3}$ components. Since $d\left(d v^{b}\right)$ must vanish, so must each of these components. We hence get the following equations:

$$
\begin{aligned}
0= & \tau_{1}^{2}-\frac{1}{21} \frac{\left(9 M^{2}+106 M+105\right) \tau_{1} \tilde{\tau}_{1}}{(1+M)^{\frac{4}{3}}}+\frac{4}{21} \frac{\left(15 M^{2}+21+28 M\right) \tilde{\tau}_{1}^{2}}{(1+M)^{\frac{5}{3}}} \\
0= & \left(\tau_{1}^{2}-5 \frac{\tau_{1} \tilde{\tau}_{1}}{(1+M)^{\frac{1}{3}}}+\frac{4 \tilde{\tau}_{1}^{2}}{(1+M)^{\frac{2}{3}}}\right) v^{a} \\
0= & \left(\tau_{1}^{2}-\frac{1}{27} \frac{\left(15 M^{2}+142 M+135\right) \tau_{1} \tilde{\tau}_{1}}{(1+M)^{\frac{4}{3}}}+\frac{4}{27} \frac{\left(21 M^{2}+40 M+27\right) \tilde{\tau}_{1}^{2}}{(1+M)^{\frac{5}{3}}}\right) g_{a b}(6.87 \mathrm{c}) \\
& +\left(\frac{8}{27} \frac{(3 M+5) \tau_{1} \tilde{\tau}_{1}}{(1+M)^{\frac{4}{3}}}-\frac{16}{27} \frac{(3 M+7) \tilde{\tau}_{1}^{2}}{(1+M)^{\frac{5}{3}}}\right) v_{a} v_{b} .
\end{aligned}
$$

Now if we contract $(6.87 \mathrm{~b})$ with $v_{a}$ and $(6.87 \mathrm{c})$ with $v^{a} v^{b}$, we get three scalar equations:

$$
\begin{aligned}
& 0=\tau_{1}^{2}-\frac{1}{21} \frac{\left(9 M^{2}+106 M+105\right) \tau_{1} \tilde{\tau}_{1}}{(1+M)^{\frac{4}{3}}}+\frac{4}{21} \frac{\left(15 M^{2}+21+28 M\right) \tilde{\tau}_{1}^{2}}{(1+M)^{\frac{5}{3}}} \\
& 0=\tau_{1}^{2}-5 \frac{\tau_{1} \tilde{\tau}_{1}}{(1+M)^{\frac{1}{3}}}+\frac{4 \tilde{\tau}_{1}^{2}}{(1+M)^{\frac{2}{3}}} \\
& 0=\tau_{1}^{2}+\frac{1}{9} \frac{\left(3 M^{2}-34 M-45\right) \tau_{1} \tilde{\tau}_{1}}{(1+M)^{\frac{4}{3}}}+\frac{4}{9} \frac{\left(3 M^{2}+4 M+9\right) \tilde{\tau}_{1}^{2}}{(1+M)^{\frac{5}{3}}} .
\end{aligned}
$$

Here our unknowns are $\tau_{1}^{2}, \tau_{1} \tilde{\tau}_{1}$ and $\tilde{\tau}_{1}^{2}$. The determinant of the system is $\frac{32}{21} \frac{M^{2}}{1+M}>0$, so the only solution is $\tau_{1}=\tilde{\tau}_{1}=0$. 
7. Concluding remarks. So far we have developed a technique for computing the deformed torsion, however there is a significant amount of work to be done to fully understand deformations of other torsion classes. We have used only a special case of the constraint (6.59), so it is likely that the full constraint will yield more information. Deformations that lie in $\Lambda_{7}^{3}$ are of course the simplest possible deformations, apart from conformal deformations, since they are defined by just a vector. The ultimate aim would be to make sense of non-infinitesimal deformations that lie in $\Lambda_{27}^{3}$. These are then defined by traceless symmetric tensors, and moreover, not all such deformations yield positive 3-forms, so extra conditions need to be imposed. On the other hand, these deformations have many more degrees of freedom than the $\Lambda_{7}^{3}$ deformations, so we could expect to get more interesting results and unlock many of the mysteries of $G_{2}$ manifolds. An ambitious program would be to try and understand which $G_{2^{-}}$ structures exist on a given manifold and what is the smallest torsion class possible on a given manifold.

\section{REFERENCES}

[1] J. BAez, The Octonions, Bull. Amer. Math. Soc. (N.S.), 39 (2002), pp. 145-205.

[2] R. L. Bryant, Metrics with exceptional holonomy, Ann. of Math. (2), 126:3 (1987), pp. 525576.

[3] R. L. BRyant, Some remarks on G_2-structures, in "Proceedings of Gökova GeometryTopology Conference 2005", pp. 75-109, Gökova Geometry/Topology Conference (GGT), Gökova, 2006.

[4] R. Cleyton and S. Ivanov, Conformal equivalence between certain geometries in dimension 6 and 7, Math. Res. Lett., 15:4 (2008), pp. 631-640.

[5] R. Cleyton and S. Ivanov, Curvature decomposition of $G_{2}$-manifolds, J. Geom. Phys., 58:10 (2008), pp. 1429-1449.

[6] M. Fernández And A. Gray, Riemannian manifolds with structure group $G_{2}$, Ann. Mat. Pura Appl. (4), 132 (1982), pp. 19-45 (1983).

[7] T. Friedrich, I. Kath, A. Moroianu, and U. Semmelmann, On nearly parallel G $_{2}$-structures, J. Geom. Phys., 23:3-4 (1997), pp. 259-286.

[8] A. Gray, Vector cross products on manifolds, Trans. Amer. Math. Soc., 141 (1969), pp. 465504.

[9] S. Grigorian, Moduli spaces of G2 manifolds, Rev. Math. Phys., 22:9 (2010), pp. 1061-1097

[10] S. Grigorian and S.-T. Yau, Local geometry of the G2 moduli space, Commun. Math. Phys., 287 (2009), pp. 459-488.

[11] N. J. Hitchin, The geometry of three-forms in six dimensions, J. Differential Geom., 55:3 (2000), pp. 547-576.

[12] D. D. Joyce, Compact manifolds with special holonomy, Oxford Mathematical Monographs, Oxford University Press, 2000.

[13] S. Karigiannis, Deformations of G_2 and Spin(7) Structures on Manifolds, Canadian Journal of Mathematics, 57 (2005), 1012.

[14] S. Karigiannis, Flows of $G_{2}$-Structures, I, Q. J. Math., 60:4 (2009), pp. 487-522.

[15] S. Karigiannis and N. C. Leung, Hodge theory for $G_{2}$-manifolds: intermediate Jacobians and Abel-Jacobi maps, Proc. Lond. Math. Soc. (3), 99:2 (2009), pp. 297-325. 
